\title{
Petrogenesis and Geodynamic Implications of Miocene Felsic Magmatic Rocks in the Wuyu Basin, Southern Gangdese Belt, Qinghai-Tibet Plateau
}

\author{
Hanzhi Chen ${ }^{1,2, *}$, Mingcai Hou ${ }^{1,3, *}$, Fuhao Xiong ${ }^{2}$, Hongwei Tang ${ }^{3,4}$ and Gangqiang Shao ${ }^{4}$ \\ 1 State Key Laboratory of Oil and Gas Reservoir Geology and Exploitation, Chengdu University of Technology, \\ Chengdu 610059, China \\ 2 College of Earth Science, Chengdu University of Technology, Chengdu 610059, China; \\ xiongfuhao2014@cdut.edu.cn \\ 3 Institute of Sedimentary Geology, Chengdu University of Technology, Chengdu 610059, China; \\ tanghongwei7116@163.com \\ 4282 Department of Sichuan Nuclear Geology, Deyang 618000, China; sgq20210415@163.com \\ * Correspondence: chenhanzhi@stu.cdut.edu.cn (H.C.); houmc@cdut.edu.cn (M.H.)
}

Citation: Chen, H.; Hou, M.;

Xiong, F.; Tang, H.; Shao, G.

Petrogenesis and Geodynamic

Implications of Miocene Felsic

Magmatic Rocks in the Wuyu Basin,

Southern Gangdese Belt, Qinghai-

Tibet Plateau. Minerals 2021, 11, 655.

https://doi.org/10.3390/min11060655

Academic Editor: Mauricio Calderón

Received: 13 April 2021

Accepted: 15 June 2021

Published: 21 June 2021

Publisher's Note: MDPI stays neutral with regard to jurisdictional claims in published maps and institutional affiliations.

Copyright: () 2021 by the authors. Licensee MDPI, Basel, Switzerland. This article is an open access article distributed under the terms and conditions of the Creative Commons Attribution (CC BY) license (https:// creativecommons.org/licenses/by/ $4.0 /)$.

\begin{abstract}
Miocene felsic magmatic rocks with high Sr/Y ratios are widely distributed throughout the Gangdese belt of southern Tibet. These provide a good opportunity to explore the magmatic process and deep dynamic mechanisms that occurred after collision between the Indo and the Asian plates. In this paper, felsic volcanic rocks from the Zongdangcun Formation in the Wuyu Basin in the central part of the southern Gangdese belt are used to disclose their origin. Zircon U-Pb geochronology analysis shows that the felsic magmatism occurred at ca. $10.3 \pm 0.2 \mathrm{Ma}$, indicating that the Zongdangcun Formation formed during the Miocene. Most of these felsic magmatic rocks plot in the rhyolite area in the TAS diagram. The rhyolite specimens from the Zongdangcun Formation have the characteristics of high $\mathrm{SiO}_{2}(>64 \%), \mathrm{K}_{2} \mathrm{O}, \mathrm{SiO}_{2}$, and $\mathrm{Sr}$ contents, a low $\mathrm{Y}$ content and a high Sr/Y ratio, and the rocks are rich in LREE and depleted in HREE, showing geochemical affinity to adakitic rocks. The rocks have an enriched $\mathrm{Sr}-\mathrm{Nd}$ isotopic composition $(\varepsilon \mathrm{Nd}(\mathrm{t})=-6.76$ to $\left.-6.68,\left({ }^{87} \mathrm{Sr} /{ }^{86} \mathrm{Sr}\right)_{i}=0.7082-0.7088\right)$, which is similar to the mixed product of the juvenile Lhasa lower continental crust and the ancient Indian crust. The Hf isotopes of zircon define a wide compositional range $(\varepsilon \mathrm{Hf}(\mathrm{t})=-4.19$ to 6.72$)$ with predominant enriched signatures. The Mioceneaged crustal thickness in southern Tibet, calculated on the basis of the $\mathrm{Sr} / \mathrm{Y}$ and $(\mathrm{La} / \mathrm{Yb})_{\mathrm{N}}$ ratios was approximately $60-80 \mathrm{~km}$, which is consistent with the thickening of the Qinghai-Tibet Plateau. The origin of Miocene felsic magmatic rocks with high Sr/Y ratios in the middle section of the Gangdese belt likely involved a partial melting of the thickened lower crust, essentially formed by the lower crust of the Lhasa block, with minor contribution from the ancient Indian crust. After comprehensively analyzing the post-collisional high Sr/Y magmatic rocks (33-8 Ma) collected from the southern margin of the Gangdese belt, we propose that the front edge tearing and segmented subduction of the Indian continental slab may be the major factor driving the east-west trending compositional changes of the Miocene adakitic rocks in southern Tibet.
\end{abstract}

Keywords: Cenozoic; Zongdangcun formation; Gangdese belt; adakitic rock

\section{Introduction}

Postcollisional Cenozoic magmatic rocks are widespread in the Qinghai-Tibetan Plateau and its surrounding areas, which provides a good window for studying the dynamic background of plateau uplift, crustal evolution, magmatism, and mineralization. The Gangdese magmatic belt is distributed along the southern margin of the Qinghai-Tibet Plateau. It is a giant Cenozoic magmatic belt created by the superposition of Neotethys oceanic lithospheric subduction and the collision of the Indian plate with the Eurasian 
plate [1]. It is the most typical composite continental magmatic arc in the world, which completely recorded the process from the subduction of the Neotethys Ocean to the collision of the Indian and the Asian plates [2-9]. Among them, the Oligocene-Miocene magmatic rocks are the products of the India-Eurasian plate collision. They mainly comprise ultrapotassic-potassic and calc-alkaline igneous rocks (Figure 1), among which, the calc-alkaline rocks generally have adakitic-like geochemical affinities [10,11]. Due to the complex genetic mechanism and magmatic dynamics of adakitic rocks, the magma source and tectonic background of the Oligocene-Miocene adakites in southern Tibet have always been controversial [12-21]. At present, most studies hypothesize that the OligoceneMiocene post-collisional adakitic rocks in southern Tibet were formed by the partial melting of the thickened lower crust beneath the Gangdese arc [12-19]. There are also many other different views on the genesis of these adakitic magmas, such as: the melting of the subducted Neotethys oceanic crust [20]; the presence of a metasomatic mantle wedge by melts derived from the subducted slab [21]; the subduction and melting of the basic lower crust of the Indian continent [15]; and the partial melting of the mantle forming water-bearing basic magma that separates and crystallizes under high pressure [22]; the mixed product of mantle-derived ultrapotassic magma and crust-derived magma [23]. Obviously, an in-depth discussion of the genetic mechanism of the Oligocene-Miocene post-collision adakitic rocks in the southern Gangdese belt is beneficial to understanding the dynamic of subduction and accretion between the Indian and Eurasian plates and is also helpful to accurately determine the evolution of the Gangdese continental crust in Cenozoic. According to the above scientific issues of the Neogene Zongdangcun Formation felsic magmatic rocks from the Wuyu Basin, Nanmulin area, southern Gangdese region are selected to perform systematic petrology, zircon U-Pb chronology, trace element geochemistry and $\mathrm{Sr}-\mathrm{Nd}-\mathrm{Hf}$ isotope. An in-depth analysis of the genetic mechanism and dynamics background of typical magmatic Oligocene-Miocene rocks on the southern margin of Gangdese was also conducted. By comprehensive comparison of the geochemical characteristics of Cenozoic post-collisional adakitic-like igneous rocks on the southern margin of Gangdese and combining them with seismic tomography data [24], the petrogenesis of Cenozoic post-collisional adakites and the spatiotemporal relationship between them and the E-W direction of the Indian plate are discussed.

\section{Geological Background and Samples}

The Neoproterozoic Qinghai-Tibet Plateau has undergone multiple subductions and accretionary orogenies of the original Tethys, Paleo-Tethys, Middle Tethys, and Neotethys suture zones, represented by the formation of the Kunlun, Longmucuo-Shuanghu suture zone, Bangong-Nujiang suture zone, and Indus-Yarlung-Tsangpo suture zone, and ophiolitic melange zone [1,2,22-26] (Figure 1A). Controlled by the Tethyan orogeny, the Kunlun block, Songpan-Ganzi block, Qiangtang block, Lhasa block, and Himalayan orogenic belt in the Qinghai-Tibet Plateau successively collided to form a giant composite collision orogen [1,27-30].

The Lhasa block, which originated from the Gondwana supercontinent, is located between the Bangong-Nujiang suture zone and the Indus-Yarlung-Tsangpo suture zone. It is composed of Precambrian crystalline basement rocks, Paleozoic Mesozoic sedimentary rocks, and Paleozoic Cenozoic magmatic rocks. The Lhasa terrane is the last one to be accreted into the Eurasian continent. The magmatic rocks of the Lhasa terrane are mainly distributed in the structural belts on the north and south sides of the terrane. The magma of the Bangong-Nujiang structural belt in the north is mainly related to the evolution of the middle Tethys Ocean. Oceanic crust subduction began at approximately $170 \mathrm{Ma}$. Following the closure of the ocean basin, the Lhasa terrane collision with the Qiangtang terrane may have started in the Late Jurassic and gone on to the mid-Cretaceous ( 99 Ma) [1,31]. The magmatic rocks in the southern Lhasa block are related to the subduction of the Neotethys Ocean and the deep subduction of the Indian plate. The Neotethys Ocean began to close at the end of the Late Cretaceous (70-65 Ma), and then the Indian plate continued to drift 
northward and finally collided with the Eurasian plate at approximately 55-50 Ma [32-34]. The Yarlung Zangbo suture zone in the south of the block was formed in this collision.
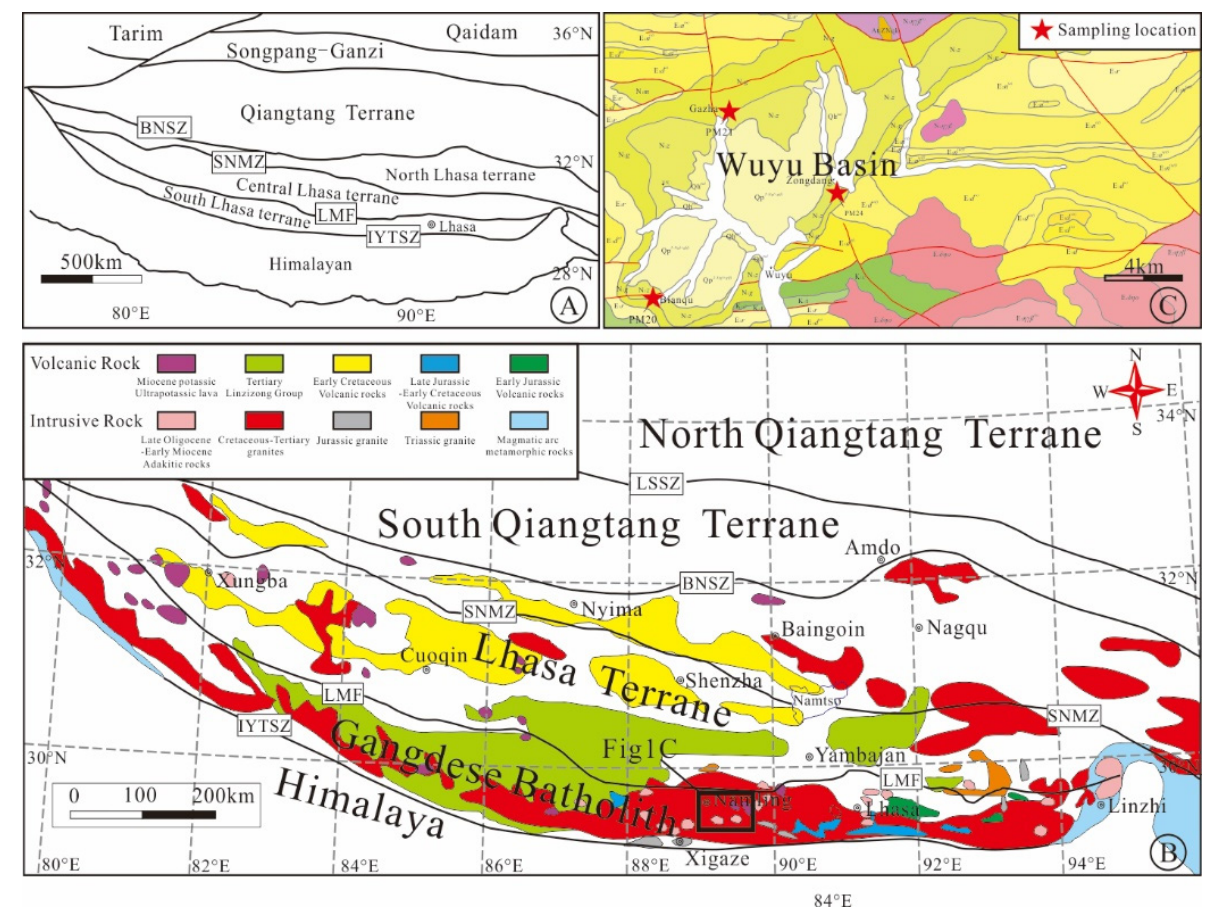

Figure 1. (A,B) Tectonic sketch of the Gangdese and Tibet Plateau, modified from [35], (C) geological map of the Wuyu Basin. LSSZ: Longmucuo-Shuanghu suture zone; BNSZ: Bangong-Nujiang suture zone; SNMZ: Shiquanhe-Namtso Ophiolite Belt zone; LMF: Lobadui-Mira fault zone; and IYTSZ: Indus-Yarlung-Tsangpo suture zone.

The Gangdese arc is the main orogen of the India-Asia collision zone, extending more than $1500 \mathrm{~km}$ along the east-west strike zone in the Lhasa terrane and is almost parallel to the Yarlung-Tsangpo suture (Figure 1A,B). The Gangdese magmatic belt has experienced long-term northward subduction of the Mesozoic Neotethys Ocean followed by strong Cenozoic magmatism during the collision between India and Eurasia [5,36-39]. It is the area with the most concentrated igneous rock, the thickest plateau crust, and the most complete collision record in the Tibetian region. Previous studies generally hypothesize that the main magmatic activities in the Gangdese magmatic belt were: (1), the Neotethys oceanic lithosphere began to subduct northward under the Lhasa block during the Middle Jurassic, forming the Middle Jurassic Yeba Formation, the Late Jurassic-Early Cretaceous Sangri Group of a subduction-related volcanic rock series, and subduction-related diorite, tonalite, granodiorite and granite suites; (2), at approximately, 65 to $50 \mathrm{Ma}$, the intrusive rocks formed during the Indian-Asian collision constitute the main body of the Gangdese belt [5,40-43]; and (3), during approximately 50-8 Ma (after the closure of the Neotethys Ocean), the igneous rock were formed after the collision in southern Tibet, comprising ultrapotassic and adakitic-like intrusions [44-49].

The Wuyu Basin is located in the middle section of the Gangdese arc and geographically located in Zhagar Village, Suojin Township, Nanmulin County of Tibet. The research focus of this paper is on the igneous rocks of the Miocene Zongdangcun Formation in the Wuyu Basin.

\section{Petrologic Characteristics}

In the southern Qinghai-Tibet Plateau, the Wuyu Basin is a relatively large part of the Cenozoic basin. The outcropping strata are the Paleocene-Eocene Linzizong Group of a mid-acid calc-alkaline volcanic rock series, the Oligocene Rigongla Formation clastic rocks 
interbedded with acid tuffs, the Miocene Mangxiang Formation of siliciclastic sediments, and the Miocene Wuyu Group, followed by a set of Pleistocene lacustrine deposits.

The Wuyu Group is divided into a lower Gazachun Formation and an upper Zongdangcun Formation. The Gazachun Formation is composed of intermediate-acid volcanic rocks and clastic rocks. The volcanic rocks of the overlying Zongdangcun Formation formed in the later stage of Wuyu Group volcanic activity, and are distributed in elliptical bodies along the periphery of the Wuyu Basin. The main volcanic rocks are represented by a set of intermediate-acid pink andesites, andesite breccias, and gray-white tuffs.

The sampling points are located in the sections around the Wuyu basin (Figure 1C). Fresh rocks less affected by hydrothermal activity were sampled. The fresh surfaces of the samples are gray and gray-green, and the weathered surfaces are gray-white. Observed under a microscope, the tuff is mainly composed of crystal (55\%) and glass fragments (45\%), and the matrix is tuffaceous. The glass components in the tuff have not been devitrified and are completely optically isotropic. The main crystals are quartz $(70 \%)$, plagioclase $(25 \%)$ biotite $(5 \%)$, and rare hornblende (Figure 2$)$. The quartz grains have a particle size of $0.01-0.5 \mathrm{~mm}$ and range from multiangular, subangular to euhedral, hexagonal, with individual sharp edges. The plagioclase has varied grain sizes, is subangular, and exhibits a faintly visible Carlsbad twin. The biotite has opaque rims.
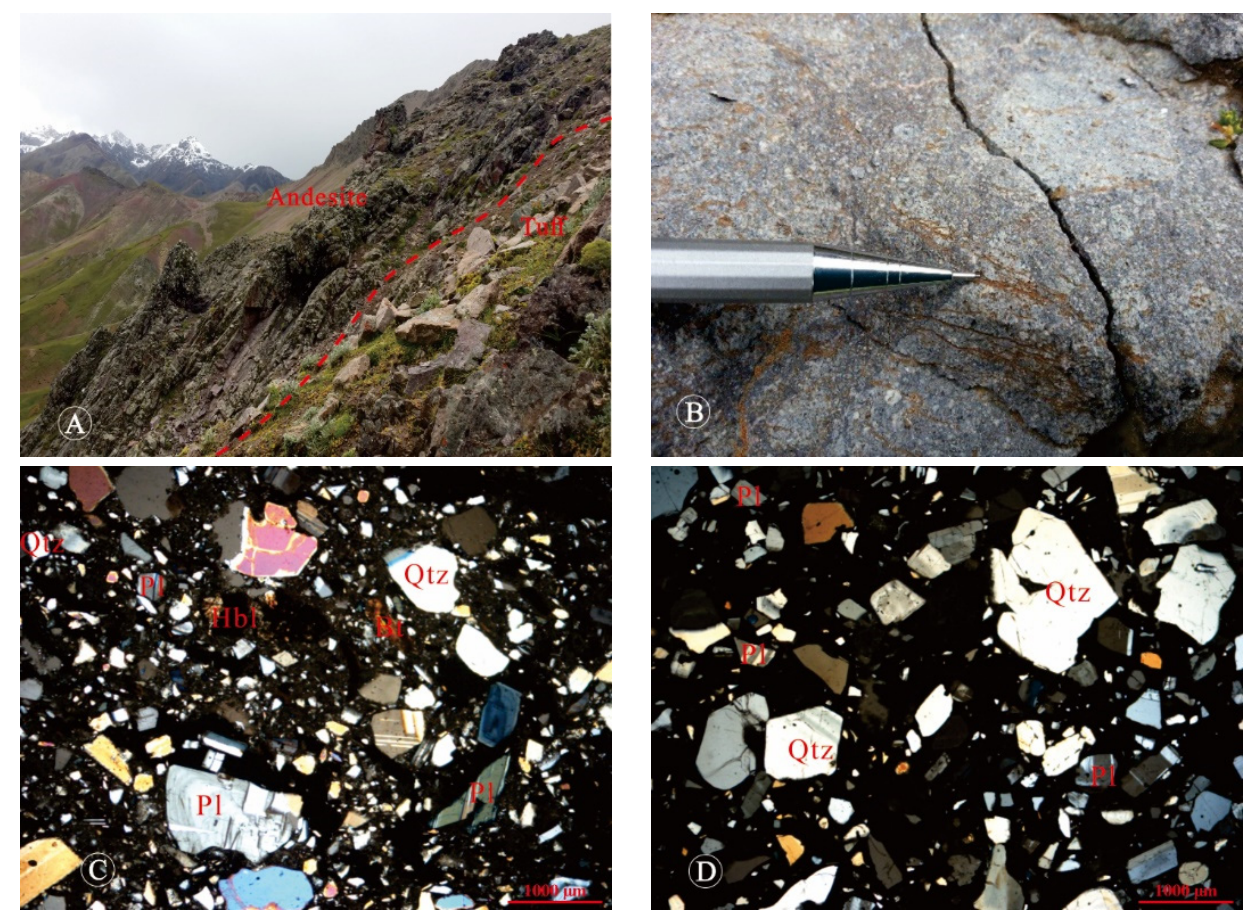

Figure 2. Macroscopic and microscopic images of the rocks of Zongdangcun Formation. (A) The boundary between tuffs and andesites; (B) Crystal-vitreous tuff outcrop of Zongdangcun Formation; (C,D) Photomicrographs of the sample at PM-20 and PM-21. Qtz: quartz, Pl: plagioclase, Bt: biotite, $\mathrm{Hbl}$ : hornblende.

\section{Analytical Methods}

\subsection{Whole-Rock Major and Trace Element Analyses}

The analysis of major, trace, and rare earth elements was performed at the State Key Laboratory of Oil and Gas Reservoir Geology and Exploitation (OGGE) of Chengdu University of Technology, Chengdu, China. Major elements were measured by XRF (X-Ray fluorescence spectrometer), and the uncertainty of this analytical method is usually $<1 \%$. Trace elements and rare earth elements (REEs) were measured by using ICP-MS, and the precision of the analysis results was better than $5-10 \%$. For the details of the analytical methods, please refer to [24]. 


\section{2. $\mathrm{Sr}$-Nd Isotopes}

The $\mathrm{Sr}$ and Nd isotopic ratios of the samples were determined by Triton plus thermal ionization mass spectrometry (Thermo Fisher Scientific, Waltham, MA, USA) (TIMS) at OGGE. The content of $\mathrm{Rb}, \mathrm{Sr}, \mathrm{Sm}$, and Nd was measured by ICP-MS, the ratio of ${ }^{87} \mathrm{Rb} /{ }^{86} \mathrm{Sr}$ and ${ }^{147} \mathrm{Sm} /{ }^{144} \mathrm{Nd}$ were calculated by them. In this experiment, NBS987 standard gave an average ${ }^{87} \mathrm{Sr} /{ }^{86} \mathrm{Sr}=0.71024 \pm 10(2 \sigma)$, mass fractionation of $\mathrm{Sr}$ isotopes was normalized to ${ }^{86} \mathrm{Sr} /{ }^{88} \mathrm{Sr}=0.1194 ; \mathrm{BCR}-2$ standard yielded an average ${ }^{143} \mathrm{Nd} /{ }^{144} \mathrm{Nd}=0.512620 \pm 2(2 \sigma)$, mass fractionation of $\mathrm{Nd}$ isotopes was normalized to ${ }^{146} \mathrm{Nd} /{ }^{144} \mathrm{Nd}=0.7219$. The measurement accuracy of $\mathrm{Rb} / \mathrm{Sr}$ and $\mathrm{Sm} / \mathrm{Nd}$ ratios is better than $0.1 \%$. For the detailed analytical methods, refer to [50].

\subsection{LA-MC-ICP-MS Zircon U-Pb Dating and Hf Isotopes}

Separation of zircon grains by heavy-liquid and magnetic methods installed in an epoxy disc and polished to expose their centers. A method of simultaneously measuring $\mathrm{U}-\mathrm{Pb}$ and Lu-Hf isotopes, as well as trace elements, was completed by Neptune MC-ICPMS and Agilent 7500a ICP-MS connected to a $193 \mathrm{~nm}$ excimer laser ablation system at the Beijing GeoAnalysis Co., Ltd. For a detailed description of the analyzer, refer to [51]. Standard zircons 91500 and GJ-1 measured after every 10 analyses, and every 30 analyses were followed by two measurements of NIST SRM 610. The relative standard deviations of reference values for 91500 were set at $2 \%$. The detailed analytical procedures are given in [52]. Offline processing of analysis data (including selection of samples and blank signals, correction of instrument sensitivity drift, element content, $\mathrm{U}-\mathrm{Pb}$ isotope ratio, and age calculation) was completed by ICP MS Data Cal software [53].

The in-situ micro area Lu-Hf isotope test in zircon was completed by the Neptune multi-receiver inductively coupled plasma mass spectrometer (Thermo Fisher Scientific, Waltham, MA, USA) and Geolas Pro laser ablation system(Coherent, Santa Clara, CA, USA). The diameter of the test beam spot was $32 \mu \mathrm{m}$. The sample aerosol ablated by the laser is transported to the mass spectrometer by helium as the carrier gas for testing. To adjust and improve the sensitivity of the instrument, argon and a small amount of nitrogen were introduced in the gas path. All test positions are the same as or close to the U-Pb dating point. The zircon standard GJ-1 is analyzed every 10 sample points for monitoring. The test accuracy of GJ-1 in this experiment is ${ }^{176} \mathrm{Hf} /{ }^{177} \mathrm{Hf}=0.282030 \pm 40(2 \sigma)$.

\section{Results}

\subsection{LA-ICP-MS Zircon U-Pb Age}

Zircon grains from one sample (PM21-Bb5) were selected for LA-ICP-MS U-Pb dating and zircon CL image acquisition. The CL images of zircon show a single color; most zircons are colorless and transparent, and the particles are euhedral-subhedra granular or columnar, with good crystallinity (Figure 3A). The cathodoluminescence image shows clear oscillatory zoning in zircon, which is typical in igneous zircons $[54,55]$. The Th content in zircon is within $252-2192 \mathrm{ppm}$, and the $U$ content is within $664-2755 \mathrm{ppm}$. There is a significant positive correlation between the two, and the Th/ $\mathrm{U}$ ratio is between 0.18 and 2.46 , which is much higher than values of metamorphic zircon (generally $<0.1$ ), and within the range of magmatic zircon [56,57]. In this experiment, 40 zircon crystals were selected from tuff sample, and the U-Pb isotopic of zircon results are shown in Table 1. The 40 zircon samples have good concordance in the U-Pb concordia diagrams. The age distribution of zircons is relatively concentrated and evenly distributed on or near the concordia curve. The results show that the U-Pb system was in a closed system after the formation of zircons, and the dating results can represent the crystallization age of the rocks. The ${ }^{206} \mathrm{~Pb} /{ }^{238} \mathrm{U}$ age span of 40 zircon samples is within a small range of 9.8-10.6 Ma, and the weighted average age is $10.3 \pm 0.2 \mathrm{a}(\mathrm{n}=40, \mathrm{MSWD}=1.10)$. 

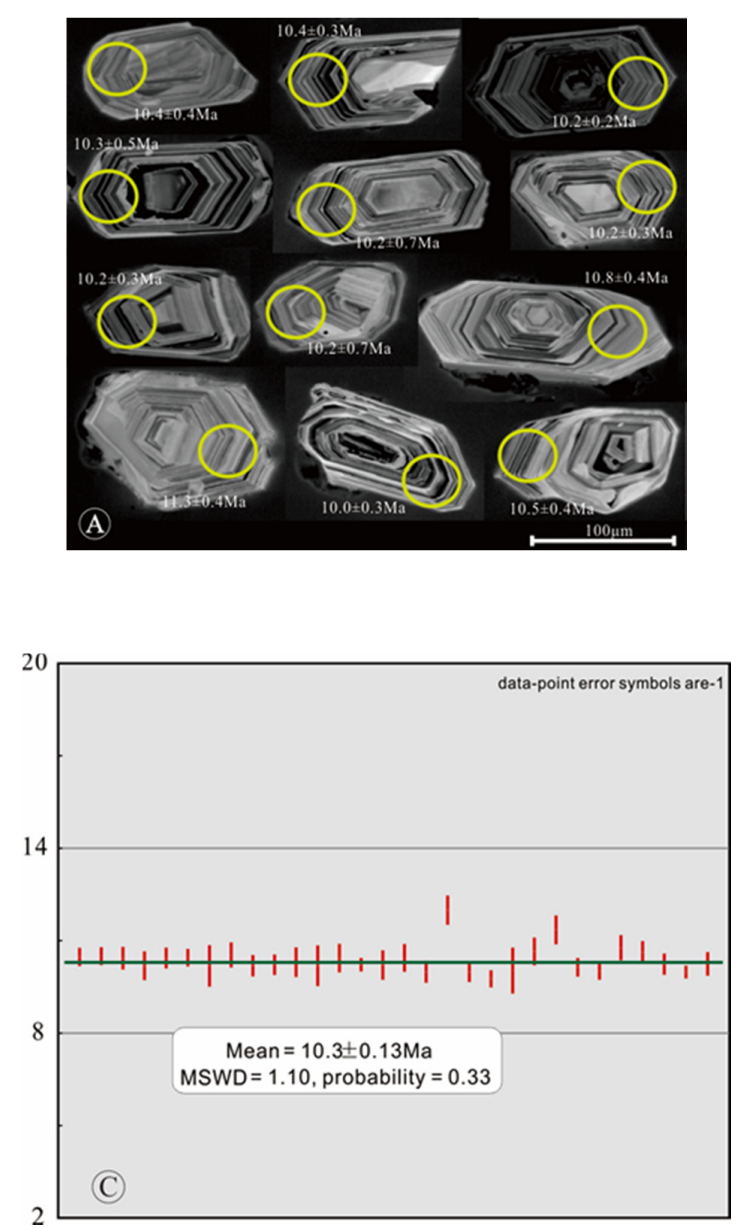
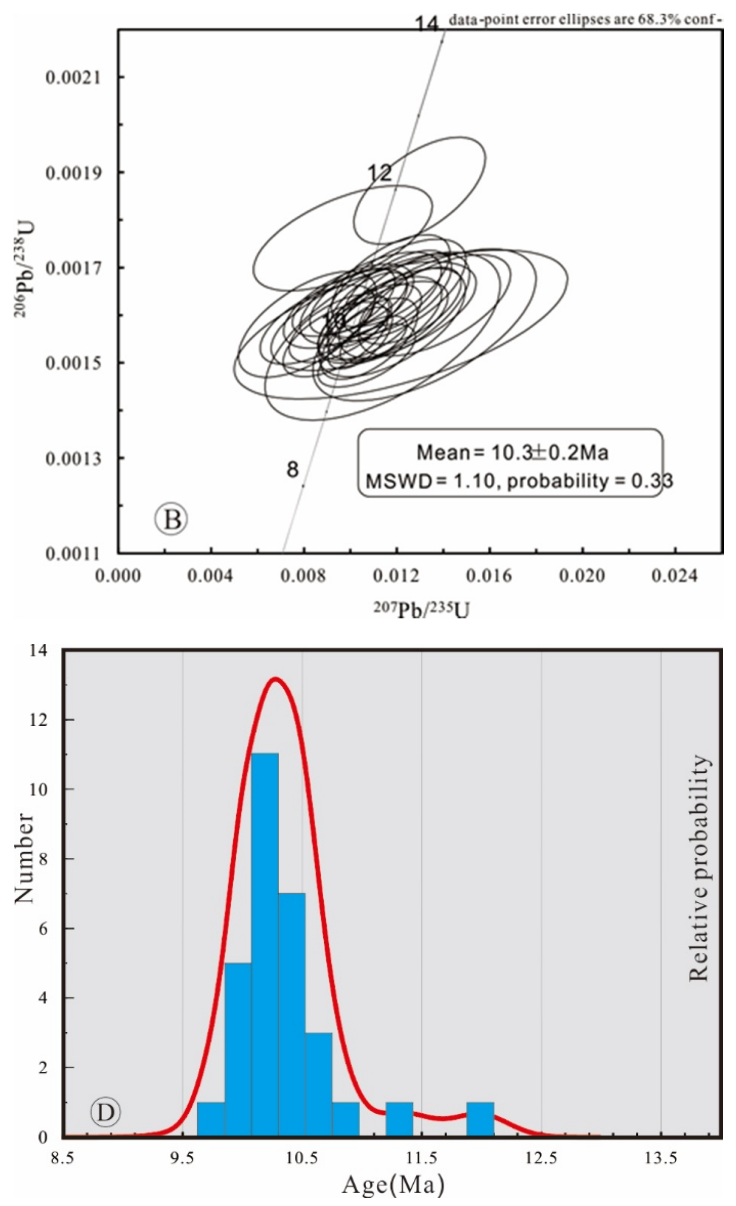

Figure 3. (A) Representative cathodoluminescence images of zircon from the tuff sample, PM21-BB5; (B) The U-Pb concordia diagrams of zircon $\mathrm{U}-\mathrm{Pb}$ for the samples; (C) Single-crystal and weighted mean zircon age U-Pb; (D) Histograms of the igneous zircons $\mathrm{U}-\mathrm{Pb}$ age from the samples.

\subsection{Whole Rock Geochemical Composition}

The major and trace element composition of 12 tuff samples from the Zongdangcun Formation collected in the Wuyu Basin of the Nanmulin area are listed in Table 2. The loss on ignition (LOI) of various samples is low, which indicates that the rocks have not been altered and that the test data are reliable. Although the chemistry of a crystal vitreous tuff does not necessarily represent the composition of the melt phase in magmas, the test results of the samples indicate that these samples can represent the magma compositions.

The compositional data show that felsic rocks of the Zongdangcun Formation are peraluminous [58], its A/CNK ratio (1.42-1.80) is greater than 1 (Figure 4C), and $\mathrm{its}^{\mathrm{SiO}_{2}}$ content is $68.41-71.37 \mathrm{wt} \%$. The $\mathrm{K}_{2} \mathrm{O}$ content is relatively high and distributed between 3.95 and $4.87 \mathrm{wt} \%$, and the samples plot into the high-potassium calcium-alkaline series [59]. The distribution range of the Rittman index $\sigma$ of the sample is $1.93-2.88<3.3$, reflecting the characteristics of calc-alkaline magmatism. The content of $\mathrm{MgO}$ is low (0.50-0.69 wt.\%); $\mathrm{Mg}^{\#}$ is less than 43.92. The samples are low in $\mathrm{TFe}_{2} \mathrm{O}_{3}$ (less than $2.38 \mathrm{wt} . \%$ ) and lowconcentration compatible elements $(\mathrm{Cr}=15.22-24.71 \mathrm{ppm} ; \mathrm{Ni}=5.33-6.49 \mathrm{ppm})$ (Table 2). The content of $\mathrm{Al}_{2} \mathrm{O}_{3}, \mathrm{CaO}, \mathrm{TiO}_{2}$, and $\mathrm{P}_{2} \mathrm{O}_{5}$ decrease with the increase of $\mathrm{SiO}_{2}$ contents (Figure 5A-D), indicating that magma differentiation. In the TAS diagram, the studied samples plot into the range of rhyolite and dacite (Figure 4A) [60], which is consistent with petrography and generally have high contents of silicon, potassium, but low contents of calcium and magnesium. 
Table 1. LA-ICP-MS zircon U-Pb age data for the crystal-vitreous tuff of the Zongdangcun Formation.

\begin{tabular}{|c|c|c|c|c|c|c|c|c|c|c|c|c|c|c|c|c|c|c|c|c|}
\hline \multirow[b]{2}{*}{ Spot } & \multirow[b]{2}{*}{$\mathrm{Pb}(\mathrm{ppm})$} & \multirow[b]{2}{*}{ Th (ppm) } & \multirow[b]{2}{*}{$\mathrm{U}(\mathrm{ppm})$} & \multirow[b]{2}{*}{$\mathrm{Th} / \mathrm{U}$} & \multicolumn{2}{|c|}{${ }^{207} \mathrm{~Pb} /{ }^{206} \mathrm{~Pb}$} & \multicolumn{2}{|c|}{${ }^{207} \mathrm{~Pb} /{ }^{235} \mathrm{U}$} & \multicolumn{2}{|c|}{${ }^{206} \mathrm{~Pb} /{ }^{238} \mathrm{U}$} & \multicolumn{2}{|c|}{${ }^{208} \mathrm{~Pb} /{ }^{232} \mathrm{Th}$} & \multicolumn{2}{|c|}{${ }^{207} \mathrm{~Pb} /{ }^{206} \mathrm{~Pb}$} & \multicolumn{2}{|c|}{${ }^{207} \mathrm{~Pb} /{ }^{235} \mathrm{U}$} & \multicolumn{2}{|c|}{${ }^{206} \mathrm{~Pb} /{ }^{238} \mathrm{U}$} & \multicolumn{2}{|c|}{${ }^{208} \mathrm{~Pb} /{ }^{232} \mathrm{Th}$} \\
\hline & & & & & Ratio & $1 \sigma$ & Ratio & $1 \sigma$ & Ratio & $1 \sigma$ & Ratio & $1 \sigma$ & $\begin{array}{l}\text { Age } \\
\text { (Ma) }\end{array}$ & $1 \sigma$ & $\begin{array}{l}\text { Age } \\
\text { (Ma) }\end{array}$ & $1 \sigma$ & $\begin{array}{l}\text { Age } \\
\text { (Ma) }\end{array}$ & $1 \sigma$ & $\begin{array}{l}\text { Age } \\
\text { (Ma) }\end{array}$ & $1 \sigma$ \\
\hline PM21-JD1-01 & 3.89 & 1385 & 1920 & 0.72 & 0.0449 & 0.0073 & 0.0099 & 0.0016 & 0.0016 & 0.0000 & 0.0004 & 0.0000 & error & & 10.0159 & 1.6394 & 10.4707 & 0.2979 & 7.8505 & 0.9331 \\
\hline PM21-JD1-02 & 5.49 & 1231 & 2755 & 0.45 & 0.0452 & 0.0057 & 0.0100 & 0.0012 & 0.0016 & 0.0000 & 0.0005 & 0.0001 & error & & 10.1242 & 1.2166 & 10.4943 & 0.2927 & 10.0574 & 1.2202 \\
\hline PM21-JD1-03 & 7.32 & 612 & 1400 & 0.44 & 0.0430 & 0.0043 & 0.0254 & 0.0026 & 0.0043 & 0.0001 & 0.0012 & 0.0001 & error & & 25.4626 & 2.5443 & 27.4721 & 0.5654 & 24.1125 & 2.4168 \\
\hline PM21-JD1-04 & 2.78 & 686 & 1373 & 0.50 & 0.0457 & 0.0090 & 0.0099 & 0.0018 & 0.0016 & 0.0001 & 0.0006 & 0.0001 & error & & 9.9801 & 1.8571 & 10.4289 & 0.3729 & 11.6017 & 1.4836 \\
\hline PM21-JD1-05 & 3.90 & 633 & 2062 & 0.31 & 0.0370 & 0.0089 & 0.0081 & 0.0020 & 0.0016 & 0.0001 & 0.0005 & 0.0001 & error & error & 8.1978 & 1.9731 & 10.1857 & 0.4704 & 9.7415 & 2.0176 \\
\hline PM21-JD1-06 & 3.84 & 1216 & 1847 & 0.66 & 0.0572 & 0.0070 & 0.0125 & 0.0015 & 0.0016 & 0.0001 & 0.0004 & 0.0001 & 498.1900 & 269.4100 & 12.6180 & 1.4625 & 10.4340 & 0.3429 & 8.4172 & 1.0141 \\
\hline PM21-JD1-07 & 7.70 & 1298 & 4021 & 0.32 & 0.0484 & 0.0045 & 0.0107 & 0.0011 & 0.0016 & 0.0000 & 0.0005 & 0.0001 & 116.7550 & 207.3750 & 10.8467 & 1.0988 & 10.4441 & 0.2861 & 9.1572 & 1.0158 \\
\hline PM21-JD1-08 & 2.56 & 1189 & 1107 & 1.07 & 0.0631 & 0.0186 & 0.0131 & 0.0032 & 0.0016 & 0.0001 & 0.0004 & 0.0001 & 722.2300 & 516.6550 & 13.2505 & 3.1765 & 10.1775 & 0.6741 & 7.8937 & 1.3825 \\
\hline PM21-JD1-09 & 2.33 & 952 & 1054 & 0.90 & 0.0485 & 0.0089 & 0.0108 & 0.0020 & 0.0016 & 0.0001 & 0.0004 & 0.0001 & 124.1600 & 381.4350 & 10.9306 & 2.0207 & 10.5332 & 0.4127 & 8.0998 & 1.0799 \\
\hline PM21-JD1-10 & 1.54 & 550 & 664 & 0.83 & 0.0594 & 0.0384 & 0.0122 & 0.0080 & 0.0017 & 0.0002 & 0.0005 & 0.0002 & 588.9150 & 994.4200 & 12.3348 & 7.9922 & 10.6310 & 1.0528 & 9.9172 & 3.0567 \\
\hline PM21-JD1-11 & 3.63 & 1255 & 1553 & 0.81 & 0.0988 & 0.0184 & 0.0213 & 0.0041 & 0.0016 & 0.0001 & 0.0005 & 0.0001 & 2000.0000 & 354.1650 & 21.4058 & 4.0366 & 10.0046 & 0.5749 & 10.6556 & 1.6824 \\
\hline PM21-JD1-12 & 4.29 & 957 & 1828 & 0.52 & 0.0665 & 0.0192 & 0.0160 & 0.0049 & 0.0016 & 0.0003 & 0.0005 & 0.0001 & 833.3300 & 633.7475 & 16.1406 & 4.9341 & 10.3387 & 1.6744 & 10.1272 & 1.7239 \\
\hline PM21-JD1-14 & 3.56 & 777 & 1798 & 0.43 & 0.0512 & 0.0073 & 0.0108 & 0.0015 & 0.0016 & 0.0001 & 0.0004 & 0.0001 & 250.0650 & 299.9700 & 10.9099 & 1.4647 & 10.2159 & 0.3366 & 8.6969 & 1.1061 \\
\hline PM21-JD1-15 & 7.72 & 2499 & 3415 & 0.73 & 0.0498 & 0.0134 & 0.0110 & 0.0029 & 0.0016 & 0.0001 & 0.0005 & 0.0001 & 187.1200 & 529.5550 & 11.0592 & 2.9282 & 10.2982 & 0.4850 & 9.4229 & 1.5143 \\
\hline PM21-JD1-16 & 6.86 & 2613 & 2998 & 0.87 & 0.0575 & 0.0250 & 0.0121 & 0.0047 & 0.0016 & 0.0001 & 0.0004 & 0.0001 & 509.3000 & 749.0250 & 12.2414 & 4.7493 & 10.1826 & 0.6638 & 8.2315 & 1.2801 \\
\hline PM21-JD1-17 & 3.36 & 994 & 1592 & 0.62 & 0.0571 & 0.0087 & 0.0123 & 0.0019 & 0.0016 & 0.0001 & 0.0004 & 0.0001 & 494.4850 & 339.6150 & 12.3906 & 1.8630 & 10.4338 & 0.4645 & 8.2867 & 1.1481 \\
\hline PM21-JD1-18 & 10.36 & 1660 & 5264 & 0.32 & 0.0475 & 0.0043 & 0.0103 & 0.0009 & 0.0016 & 0.0000 & 0.0004 & 0.0001 & 76.0200 & 199.9700 & 10.4404 & 0.9241 & 10.2150 & 0.2200 & 8.4395 & 1.0328 \\
\hline PM21-JD1-19 & 3.38 & 917 & 1556 & 0.59 & 0.0553 & 0.0101 & 0.0117 & 0.0021 & 0.0016 & 0.0001 & 0.0004 & 0.0001 & 433.3800 & 357.3600 & 11.8534 & 2.0950 & 10.2050 & 0.4820 & 8.8238 & 1.4958 \\
\hline PM21-JD1-20 & 2.17 & 671 & 942 & 0.71 & 0.0643 & 0.0110 & 0.0136 & 0.0022 & 0.0016 & 0.0001 & 0.0005 & 0.0001 & 753.7100 & 367.1025 & 13.6885 & 2.1611 & 10.4385 & 0.4521 & 10.1665 & 1.5644 \\
\hline PM21-JD1-21 & 3.76 & 913 & 1909 & 0.48 & 0.0510 & 0.0068 & 0.0108 & 0.0014 & 0.0016 & 0.0001 & 0.0004 & 0.0001 & 238.9550 & 281.4550 & 10.8638 & 1.3906 & 9.9878 & 0.3598 & 7.5948 & 1.2334 \\
\hline PM21-JD1-22 & 5.78 & 896 & 2996 & 0.30 & 0.0629 & 0.0135 & 0.0136 & 0.0031 & 0.0015 & 0.0001 & 0.0006 & 0.0001 & 705.5650 & 468.4775 & 13.6672 & 3.0616 & 9.9708 & 0.4021 & 11.7081 & 2.8853 \\
\hline PM21-JD1-23 & 3.40 & 473 & 1545 & 0.31 & 0.0503 & 0.0067 & 0.0129 & 0.0019 & 0.0019 & 0.0001 & 0.0006 & 0.0001 & 209.3300 & 290.7100 & 13.0450 & 1.8706 & 11.9857 & 0.4758 & 12.5874 & 1.6950 \\
\hline PM21-JD1-24 & 6.41 & 702 & 3492 & 0.20 & 0.0440 & 0.0070 & 0.0093 & 0.0015 & 0.0015 & 0.0001 & 0.0004 & 0.0001 & error & & 9.4110 & 1.4967 & 9.9842 & 0.3320 & 8.4437 & 1.6980 \\
\hline PM21-JD1-25 & 6.96 & 1162 & 3939 & 0.30 & 0.0500 & 0.0053 & 0.0099 & 0.0010 & 0.0015 & 0.0000 & 0.0004 & 0.0001 & 194.5250 & 238.8550 & 10.0044 & 1.0150 & 9.3565 & 0.2568 & 8.1900 & 1.0642 \\
\hline PM21-JD1-26 & 5.98 & 1576 & 3036 & 0.52 & 0.0518 & 0.0072 & 0.0107 & 0.0014 & 0.0015 & 0.0000 & 0.0004 & 0.0001 & 275.9900 & 288.8600 & 10.7579 & 1.3883 & 9.7602 & 0.2804 & 9.0247 & 1.1651 \\
\hline PM21-JD1-27 & 3.35 & 2230 & 904 & 2.47 & 0.1482 & 0.0517 & 0.0331 & 0.0108 & 0.0016 & 0.0002 & 0.0005 & 0.0001 & 2325.0050 & 629.4700 & 33.0804 & 10.6336 & 10.6060 & 1.2051 & 10.4401 & 1.2160 \\
\hline
\end{tabular}


Table 1. Cont.

\begin{tabular}{|c|c|c|c|c|c|c|c|c|c|c|c|c|c|c|c|c|c|c|c|c|}
\hline \multirow[b]{2}{*}{ Spot } & \multirow[b]{2}{*}{$\mathrm{Pb}(\mathrm{ppm})$} & \multirow[b]{2}{*}{ Th (ppm) } & \multirow[b]{2}{*}{$\mathrm{U}(\mathrm{ppm})$} & \multirow[b]{2}{*}{$\mathrm{Th} / \mathrm{U}$} & \multicolumn{2}{|c|}{${ }^{207} \mathrm{~Pb} /{ }^{206} \mathrm{~Pb}$} & \multicolumn{2}{|c|}{${ }^{207} \mathrm{~Pb} /{ }^{235} \mathrm{U}$} & \multicolumn{2}{|c|}{${ }^{206} \mathrm{~Pb} /{ }^{238} \mathrm{U}$} & \multicolumn{2}{|c|}{${ }^{208} \mathrm{~Pb} /{ }^{232} \mathrm{Th}$} & \multicolumn{2}{|c|}{${ }^{207} \mathrm{~Pb} /{ }^{206} \mathrm{~Pb}$} & \multicolumn{2}{|c|}{${ }^{207} \mathrm{~Pb} /{ }^{235} \mathrm{U}$} & \multicolumn{2}{|c|}{${ }^{206} \mathrm{~Pb} /{ }^{238} \mathrm{U}$} & \multicolumn{2}{|c|}{${ }^{208} \mathrm{~Pb} /{ }^{232} \mathrm{Th}$} \\
\hline & & & & & Ratio & $1 \sigma$ & Ratio & $1 \sigma$ & Ratio & $1 \sigma$ & Ratio & $1 \sigma$ & $\begin{array}{l}\text { Age } \\
\text { (Ma) }\end{array}$ & $1 \sigma$ & $\begin{array}{l}\text { Age } \\
\text { (Ma) }\end{array}$ & $1 \sigma$ & $\begin{array}{l}\text { Age } \\
\text { (Ma) }\end{array}$ & $1 \sigma$ & $\begin{array}{l}\text { Age } \\
\text { (Ma) }\end{array}$ & $1 \sigma$ \\
\hline PM21-JD1-28 & 5.06 & 3010 & 2030 & 1.48 & 0.0552 & 0.0174 & 0.0113 & 0.0033 & 0.0016 & 0.0001 & 0.0004 & 0.0001 & 420.4200 & 582.3550 & 11.4517 & 3.3615 & 10.0263 & 0.7478 & 8.2309 & 1.6536 \\
\hline PM21-JD1-29 & 11.69 & 7367 & 3593 & 2.05 & 0.0512 & 0.0058 & 0.0130 & 0.0015 & 0.0019 & 0.0001 & 0.0005 & 0.0001 & 250.0650 & 249.9750 & 13.0651 & 1.4953 & 11.9324 & 0.4515 & 10.5252 & 1.5226 \\
\hline PM21-JD1-30 & 2.76 & 822 & 1309 & 0.63 & 0.0537 & 0.0088 & 0.0119 & 0.0020 & 0.0017 & 0.0001 & 0.0004 & 0.0001 & 366.7200 & 327.7350 & 12.0370 & 1.9872 & 10.6484 & 0.4546 & 8.1357 & 1.9308 \\
\hline PM21-JD1-31 & 1.69 & 372 & 788 & 0.47 & 0.0407 & 0.0110 & 0.0096 & 0.0025 & 0.0018 & 0.0001 & 0.0005 & 0.0001 & error & & 9.7208 & 2.5474 & 11.3482 & 0.4683 & 10.2954 & 1.8062 \\
\hline PM21-JD1-32 & 3.92 & 1980 & 1638 & 1.21 & 0.0433 & 0.0065 & 0.0095 & 0.0014 & 0.0016 & 0.0000 & 0.0004 & 0.0000 & error & & 9.5684 & 1.4360 & 10.1311 & 0.3060 & 8.0332 & 0.8119 \\
\hline PM21-JD1-33 & 9.43 & 2114 & 4918 & 0.43 & 0.0494 & 0.0049 & 0.0104 & 0.0009 & 0.0016 & 0.0000 & 0.0003 & 0.0000 & 164.9000 & 224.0400 & 10.4764 & 0.9243 & 10.0015 & 0.2749 & 7.0168 & 0.8464 \\
\hline PM21-JD1-34 & 6.85 & 2257 & 1498 & 1.51 & 0.0484 & 0.0063 & 0.0196 & 0.0025 & 0.0029 & 0.0001 & 0.0007 & 0.0001 & 120.4600 & 277.7400 & 19.6934 & 2.5385 & 18.9102 & 0.6182 & 14.1629 & 1.3537 \\
\hline PM21-JD1-35 & 2.70 & 253 & 1423 & 0.18 & 0.0517 & 0.0073 & 0.0117 & 0.0017 & 0.0017 & 0.0001 & 0.0006 & 0.0001 & 333.3900 & 231.4600 & 11.8002 & 1.6860 & 10.7698 & 0.4094 & 11.5635 & 1.8038 \\
\hline PM21-JD1-36 & 7.19 & 1334 & 3669 & 0.36 & 0.0678 & 0.0101 & 0.0143 & 0.0022 & 0.0016 & 0.0001 & 0.0004 & 0.0001 & 861.1050 & 312.9475 & 14.4451 & 2.1872 & 10.0135 & 0.4200 & 8.4582 & 1.6327 \\
\hline PM21-JD1-37 & 3.65 & 1204 & 1656 & 0.73 & 0.0543 & 0.0076 & 0.0119 & 0.0016 & 0.0016 & 0.0001 & 0.0004 & 0.0001 & 383.3850 & 319.2450 & 12.0543 & 1.5564 & 10.6156 & 0.3727 & 8.4842 & 1.1241 \\
\hline PM21-JD1-38 & 4.09 & 1716 & 1856 & 0.92 & 0.0551 & 0.0089 & 0.0116 & 0.0017 & 0.0016 & 0.0001 & 0.0004 & 0.0000 & 416.7150 & 364.7700 & 11.6668 & 1.7216 & 10.2338 & 0.3484 & 7.1273 & 0.7010 \\
\hline PM21-JD1-39 & 11.35 & 5410 & 5006 & 1.08 & 0.0515 & 0.0044 & 0.0109 & 0.0010 & 0.0015 & 0.0000 & 0.0004 & 0.0000 & 264.8800 & 198.1225 & 11.0475 & 0.9592 & 9.9779 & 0.2100 & 7.3946 & 0.6281 \\
\hline
\end{tabular}

Error: Due to the small size of some zircon crystals, the amount of laser ablation is not enough to obtain effective isotope and age information.

Table 2. Major and trace element analysis of adakitic rock for the Zongdangcun formation.

\begin{tabular}{|c|c|c|c|c|c|c|c|c|c|c|c|c|}
\hline Simple & PM20-10-Bb1 & PM21-10-Bb1 & PM24-1-Bb1 & PM24-4-Bb1 & PM24-5-Bb1 & PM21-Bb2 & PM21-Bb3 & PM21-Bb4 & PM21-Bb5 * & PM21-Bb8 & PM21-4-Bb1 & PM21-6-Bb1 \\
\hline $\mathrm{SiO}_{2}(\mathrm{wt} \%)$ & 69.13 & 69.18 & 68.41 & 68.57 & 68.27 & 71.01 & 71.37 & 70.02 & 68.95 & 69.17 & 68.90 & 69.32 \\
\hline $\mathrm{TiO}_{2}$ & 0.33 & 0.41 & 0.36 & 0.35 & 0.33 & 0.31 & 0.28 & 0.30 & 0.31 & 0.30 & 0.32 & 0.28 \\
\hline $\mathrm{Al}_{2} \mathrm{O}_{3} \%$ & 15.33 & 15.26 & 15.54 & 15.87 & 15.17 & 15.09 & 15.03 & 15.29 & 15.01 & 14.93 & 15.41 & 14.98 \\
\hline $\mathrm{TFe}_{2} \mathrm{O}_{3} \%$ & 2.16 & 2.33 & 2.23 & 2.30 & 2.31 & 1.83 & 1.86 & 1.57 & 1.77 & 1.90 & 2.38 & 2.07 \\
\hline $\mathrm{MnO}$ & 0.06 & 0.03 & 0.03 & 0.03 & 0.04 & 0.03 & 0.03 & 0.04 & 0.03 & 0.03 & 0.03 & 0.04 \\
\hline $\mathrm{MgO} \%$ & 0.58 & 0.50 & 0.66 & 0.68 & 0.62 & 0.57 & 0.52 & 0.69 & 0.56 & 0.60 & 0.53 & 0.58 \\
\hline $\mathrm{CaO} \%$ & 1.92 & 2.27 & 2.40 & 1.94 & 2.72 & 1.86 & 1.68 & 2.11 & 2.13 & 2.07 & 1.87 & 1.75 \\
\hline $\mathrm{Na}_{2} \mathrm{O} \%$ & 3.80 & 3.58 & 3.66 & 3.61 & 3.59 & 3.46 & 3.56 & 2.88 & 2.91 & 2.91 & 3.53 & 4.03 \\
\hline $\mathrm{K}_{2} \mathrm{O} \%$ & 4.87 & 4.03 & 4.71 & 4.87 & 4.40 & 4.16 & 4.55 & 4.35 & 4.44 & 4.52 & 4.10 & 3.95 \\
\hline $\mathrm{P}_{2} \mathrm{O}_{5}$ & 0.12 & 0.12 & 0.13 & 0.11 & 0.13 & 0.12 & 0.12 & 0.10 & 0.14 & 0.12 & 0.12 & 0.12 \\
\hline
\end{tabular}


Table 2. Cont.

\begin{tabular}{|c|c|c|c|c|c|c|c|c|c|c|c|c|}
\hline Simple & PM20-10-Bb1 & PM21-10-Bb1 & PM24-1-Bb1 & PM24-4-Bb1 & PM24-5-Bb1 & PM21-Bb2 & PM21-Bb3 & PM21-Bb4 & PM21-Bb5 * & PM21-Bb8 & PM21-4-Bb1 & PM21-6-Bb1 \\
\hline LOI\% & 1.50 & 2.14 & 1.70 & 1.47 & 2.62 & 0.90 & 0.35 & 2.40 & 2.32 & 2.43 & 1.22 & 1.14 \\
\hline Mg\# & 32.46 & 27.71 & 34.51 & 34.57 & 32.31 & 35.70 & 33.26 & 43.93 & 36.06 & 36.02 & 28.42 & 33.31 \\
\hline $\mathrm{Rb}(\mathrm{ppm})$ & 291.86 & 221.19 & 213.43 & 263.49 & 187.26 & 203.00 & 222.00 & 210.00 & 214.00 & 220.00 & 195.00 & 180.00 \\
\hline $\mathrm{Ba}$ & 1068.93 & 1055.32 & 1144.15 & 1107.62 & 1101.29 & 924.00 & 900.00 & 886.00 & 891.00 & 875.00 & 942.00 & 854.00 \\
\hline Th & 52.69 & 47.88 & 58.41 & 52.76 & 50.42 & 48.40 & 45.20 & 48.90 & 48.00 & 48.20 & 48.20 & 46.50 \\
\hline $\mathrm{U}$ & 6.37 & 4.61 & 6.07 & 5.52 & 5.79 & 3.54 & 4.20 & 7.70 & 7.56 & 8.01 & 2.07 & 3.82 \\
\hline $\mathrm{Ta}$ & 1.39 & 0.77 & 0.95 & 1.31 & 0.87 & 1.04 & 1.02 & 0.90 & 1.14 & 1.10 & 0.92 & 0.79 \\
\hline $\mathrm{Nb}$ & 15.06 & 11.23 & 11.48 & 13.48 & 10.17 & 11.30 & 11.10 & 11.80 & 10.40 & 11.60 & 10.60 & 9.94 \\
\hline $\mathrm{Sr}$ & 477.74 & 604.07 & 548.25 & 498.35 & 522.19 & 604.00 & 572.00 & 732.00 & 728.00 & 778.00 & 620.00 & 574.00 \\
\hline $\mathrm{P}$ & 512.10 & 518.30 & 581.20 & 481.90 & 547.90 & 515.02 & 523.75 & 449.55 & 589.22 & 523.75 & 506.29 & 519.39 \\
\hline $\mathrm{Zr}$ & 81.76 & 105.10 & 94.58 & 73.96 & 89.56 & 168.00 & 162.00 & 162.00 & 166.00 & 169.00 & 184.00 & 197.00 \\
\hline $\mathrm{Hf}$ & 3.79 & 3.79 & 3.48 & 3.23 & 3.29 & 4.50 & 4.40 & 4.50 & 4.40 & 4.50 & 5.00 & 7.60 \\
\hline $\mathrm{Y}$ & 11.43 & 9.96 & 10.39 & 9.32 & 9.58 & 11.80 & 9.95 & 11.00 & 11.10 & 11.70 & 11.60 & 10.60 \\
\hline Sc & 2.49 & 2.15 & 2.55 & 2.48 & 2.29 & 2.37 & 1.77 & 2.53 & 2.34 & 2.15 & 2.34 & 2.42 \\
\hline $\mathrm{V}$ & 30.02 & 37.70 & 34.57 & 33.75 & 31.10 & 33.02 & 30.78 & 30.03 & 32.17 & 34.43 & 30.11 & 31.15 \\
\hline $\mathrm{Cr}$ & 24.71 & 21.09 & 18.96 & 16.36 & 18.35 & 21.74 & 23.81 & 19.33 & 16.46 & 17.42 & 15.22 & 17.13 \\
\hline Co & 3.57 & 2.76 & 3.71 & 4.08 & 4.54 & 3.65 & 3.64 & 4.22 & 3.16 & 3.77 & 2.98 & 4.32 \\
\hline $\mathrm{Ni}$ & 5.36 & 5.41 & 5.86 & 5.33 & 6.49 & 5.41 & 5.33 & 5.49 & 5.46 & 5.93 & 6.02 & 6.21 \\
\hline $\mathrm{La}$ & 59.54 & 59.03 & 62.01 & 54.72 & 58.89 & 57.60 & 49.00 & 51.30 & 49.40 & 52.60 & 55.00 & 52.40 \\
\hline $\mathrm{Ce}$ & 98.99 & 95.97 & 99.17 & 85.33 & 90.82 & 106.00 & 90.00 & 95.90 & 92.40 & 97.60 & 102.00 & 94.20 \\
\hline $\operatorname{Pr}$ & 10.90 & 10.42 & 11.12 & 9.88 & 10.12 & 11.00 & 9.50 & 10.40 & 9.96 & 10.70 & 10.80 & 10.20 \\
\hline $\mathrm{Sm}$ & 5.79 & 5.33 & 5.80 & 4.84 & 5.07 & 5.49 & 4.67 & 5.23 & 5.06 & 5.38 & 5.40 & 5.12 \\
\hline $\mathrm{Eu}$ & 1.21 & 1.33 & 1.35 & 1.35 & 1.34 & 1.15 & 1.08 & 1.10 & 1.14 & 1.17 & 1.19 & 1.12 \\
\hline $\mathrm{Gd}$ & 4.35 & 4.28 & 4.55 & 3.82 & 3.92 & 4.70 & 4.03 & 4.48 & 4.30 & 4.66 & 4.77 & 4.39 \\
\hline $\mathrm{Tb}$ & 0.59 & 0.55 & 0.56 & 0.52 & 0.49 & 0.52 & 0.44 & 0.50 & 0.48 & 0.51 & 0.52 & 0.49 \\
\hline Dy & 2.02 & 1.86 & 1.91 & 1.75 & 1.64 & 2.30 & 1.90 & 2.24 & 2.13 & 2.24 & 2.31 & 2.18 \\
\hline Ho & 0.36 & 0.33 & 0.34 & 0.31 & 0.30 & 0.37 & 0.30 & 0.36 & 0.35 & 0.36 & 0.36 & 0.36 \\
\hline Er & 1.25 & 1.13 & 1.23 & 1.13 & 1.12 & 1.16 & 1.00 & 1.10 & 1.08 & 1.14 & 1.16 & 1.08 \\
\hline $\mathrm{Tm}$ & 0.16 & 0.13 & 0.15 & 0.13 & 0.12 & 0.16 & 0.14 & 0.16 & 0.16 & 0.16 & 0.15 & 0.14 \\
\hline $\mathrm{Yb}$ & 0.95 & 0.79 & 0.82 & 0.80 & 0.75 & 1.02 & 0.87 & 0.94 & 1.00 & 1.06 & 0.98 & 0.90 \\
\hline $\mathrm{Lu}$ & 0.16 & 0.13 & 0.16 & 0.15 & 0.14 & 0.14 & 0.12 & 0.14 & 0.14 & 0.15 & 0.14 & 0.13 \\
\hline$\Sigma R E E$ & 225.60 & 218.21 & 229.55 & 198.79 & 210.33 & 229.71 & 195.65 & 209.45 & 202.00 & 214.53 & 222.18 & 207.61 \\
\hline LREE & 215.76 & 209.02 & 219.84 & 190.18 & 201.84 & 219.34 & 186.85 & 199.53 & 192.36 & 204.25 & 211.79 & 197.94 \\
\hline HREE & 9.85 & 9.19 & 9.71 & 8.61 & 8.49 & 10.37 & 8.80 & 9.92 & 9.64 & 10.28 & 10.39 & 9.67 \\
\hline LREE/HREE & 21.91 & 22.75 & 22.64 & 22.09 & 23.77 & 21.15 & 21.23 & 20.11 & 19.95 & 19.87 & 20.38 & 20.47 \\
\hline $\mathrm{La}_{\mathrm{N}} / \mathrm{Yb}_{\mathrm{N}}$ & 44.77 & 53.80 & 54.18 & 49.12 & 56.25 & 40.51 & 40.40 & 39.15 & 35.43 & 35.59 & 40.26 & 41.76 \\
\hline$\delta \mathrm{Eu}$ & 0.71 & 0.82 & 0.78 & 0.93 & 0.88 & 0.67 & 0.74 & 0.68 & 0.73 & 0.70 & 0.70 & 0.70 \\
\hline$\delta \mathrm{Ce}$ & 0.88 & 0.87 & 0.86 & 0.83 & 0.84 & 0.97 & 0.96 & 0.96 & 0.96 & 0.95 & 0.96 & 0.94 \\
\hline
\end{tabular}



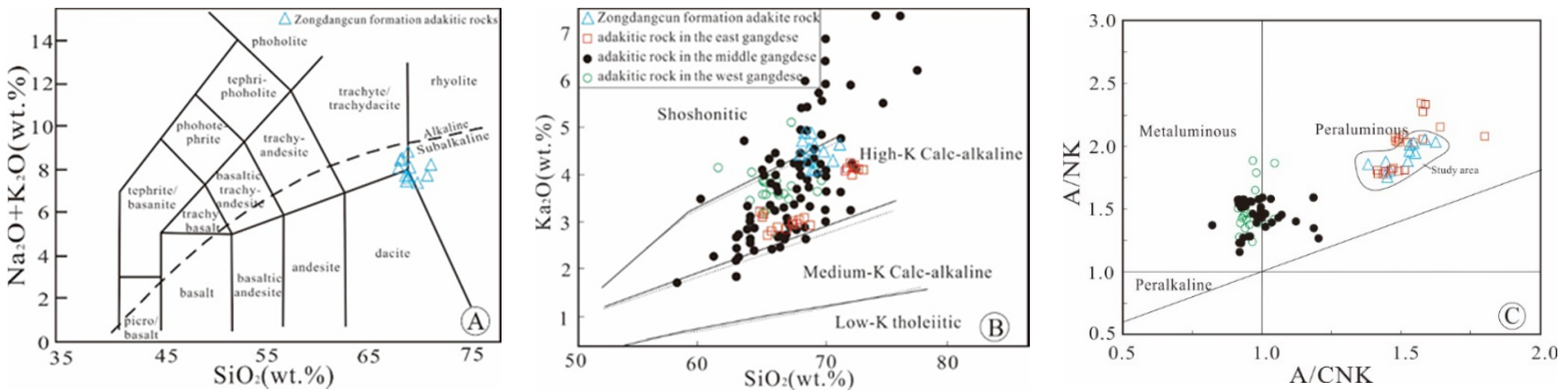

Figure 4. (A) TAS classification diagram [60]. (B) $\mathrm{SiO}_{2}-\mathrm{K}_{2} \mathrm{O}$ plot for tuff [59]. (C) A/CNKvs. A/NK diagram [58] Data from $[11,12,19,44,61]$.
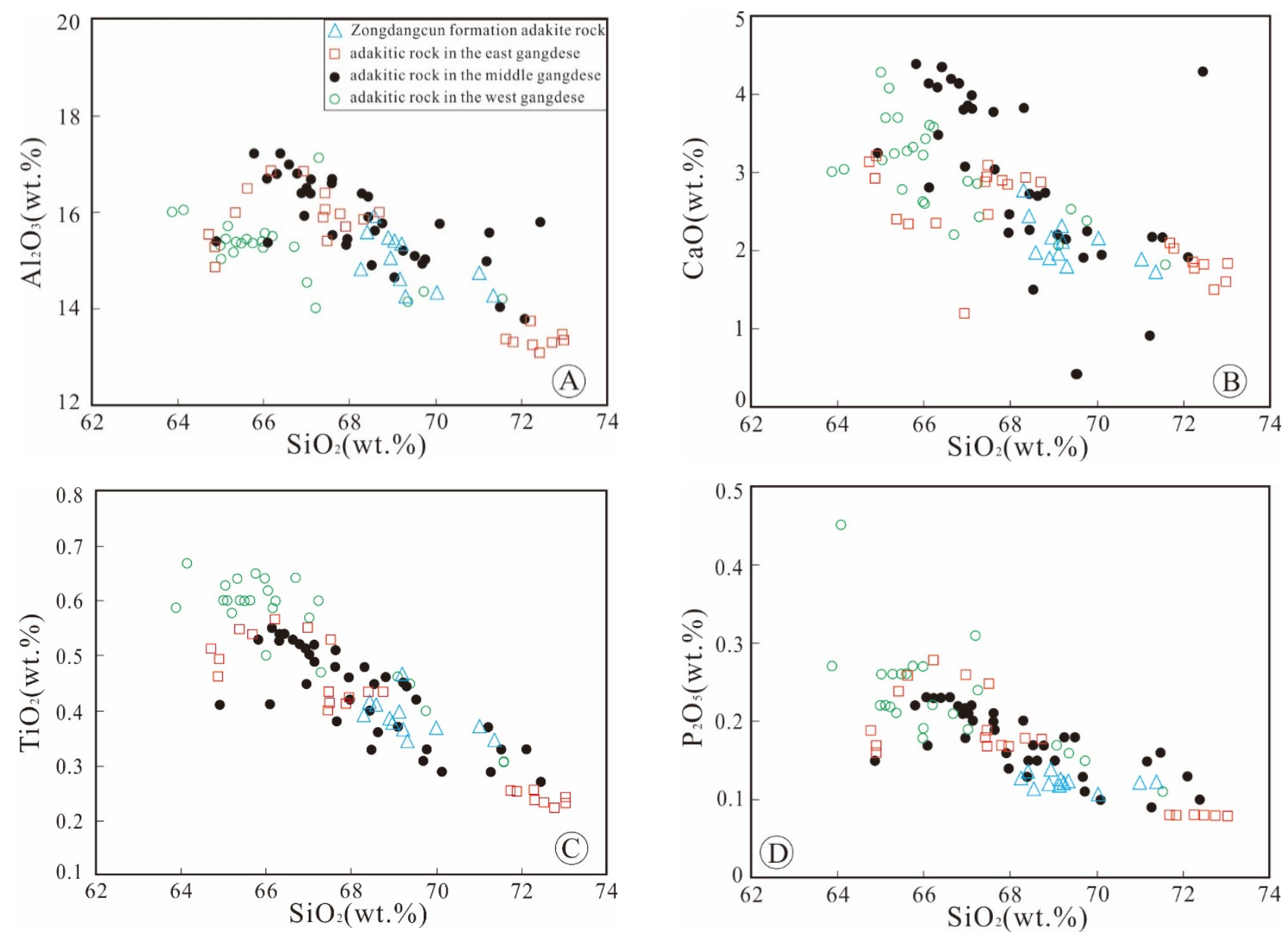

Figure 5. Harker variation diagrams (A-D), the major element changes of Miocene adakites in Lhasa blocks are shown. Data from the same source as Figure 4.

The Zongdangcun tuffs in the Wuyu Basin have similar trace element composition. The samples are enriched in LILE [62] but depleted in HFSE (Figure 6). High Sr (477-778 ppm) and low Y (9.32-11.80 ppm) concentrations lead to high Sr/Y ratios (41.8-66.5) and slight negative Eu anomalies (0.67-0.93); strong negative $\mathrm{Nb}, \mathrm{Ta}, \mathrm{P}, \mathrm{Ti}, \mathrm{Sr}$ anomalies and positive $\mathrm{Zr}$ and $\mathrm{Hf}$ anomalies are shown in the primitive mantle-normalized element spider diagram (Figure 6). In addition, the samples show a right leaning pattern of LREE enrichment and HREE depletion $\left((\mathrm{La} / \mathrm{Yb})_{\mathrm{N}}>26.18\right)$. The contents of major and 
trace elements in the samples are consistent with the geochemical characteristics of adakitic rocks [63-66]. In Figure 7, all samples plot in the adakitic area.
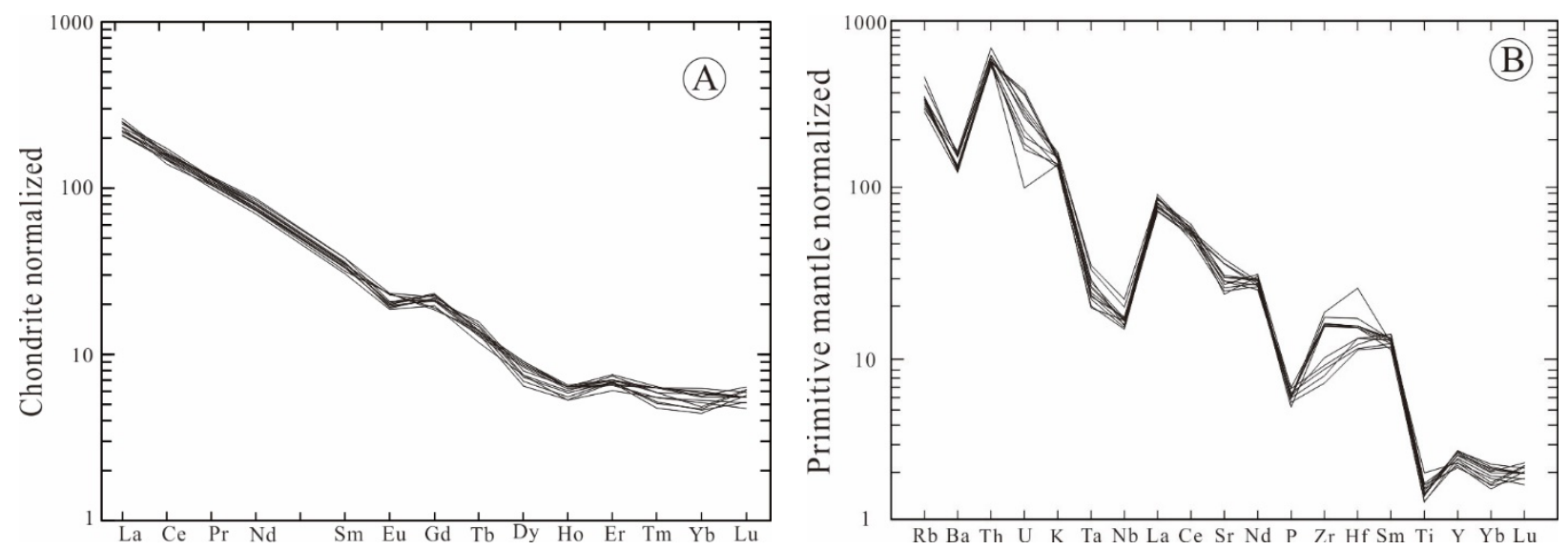

Figure 6. (A) Chondrite-normalized rare earth element diagrams; (B) Primitive mantle-normalized element spider diagram. Normalizing values from [67].
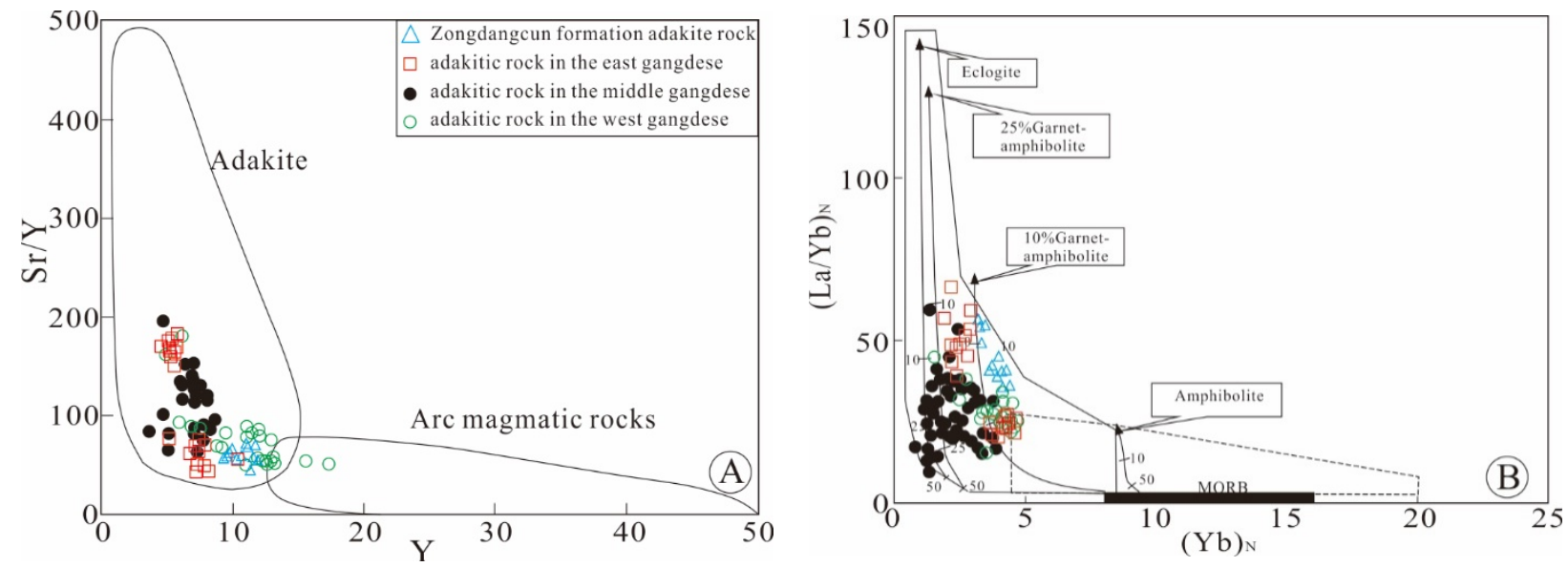

Figure 7. (A) Diagrams showing the relationships of Sr/Y vs. Y [66]; (B) (La/Yb) $)_{\mathrm{N}}$ vs. $\mathrm{Yb}_{\mathrm{N}}$ [68] for Miocene magmatic rocks from Gangdese batholith, data from the same source as Figure 4.

\subsection{Sr-Nd (Bulk Rock) and Hf (Zircon) Isotopic Data}

The initial Sr-Nd isotopic compositions of three samples (PM20-10-Bb1, PM21-10$\mathrm{Bb} 1, \mathrm{PM} 24-1-\mathrm{Bb} 1)$ were calculated by using zircon ${ }^{206} \mathrm{~Pb} /{ }^{238} \mathrm{U}$ weighted average ages $(10.3 \pm 0.2 \mathrm{Ma})$ (Table 3). They are characterized by a relatively uniform $\varepsilon \mathrm{Nd}(\mathrm{t})$ value $(-6.76--6.68)$ and an almost constant initial $\left({ }^{87} \mathrm{Sr} /{ }^{86} \mathrm{Sr}\right)_{\mathrm{i}}$ isotope ratio $(0.7082-0.7088)$. The age of the single-stage $\mathrm{Nd}$ model is $\mathrm{T}_{\mathrm{DM}}=1040-1057 \mathrm{Ma}$, and the age of the two-stage $\mathrm{Nd}$ model is $\mathrm{T}_{\mathrm{DM} 2}=1371-1377 \mathrm{Ma}$.

Table 3. Whole rock Sr-Nd isotopic data for the silicic tuffs of the Zongdangcun Formation.

\begin{tabular}{|c|c|c|c|c|c|c|c|c|c|c|c|c|}
\hline Sample & $\begin{array}{c}\mathrm{Rb} \\
\mathrm{ppm}\end{array}$ & Sr ppm & ${ }^{87} \mathrm{Rb} /{ }^{86} \mathrm{Sr}$ & ${ }^{87} \mathrm{Sr} /{ }^{86} \mathrm{Sr}$ & $\left({ }^{87} \mathrm{Sr} /{ }^{86} \mathrm{Sr}\right) \mathrm{i}$ & $\begin{array}{c}\text { Sm } \\
\text { ppm }\end{array}$ & $\begin{array}{c}\text { Nd } \\
\text { ppm }\end{array}$ & ${ }^{147} \mathrm{Sm} /{ }^{144} \mathrm{Nd}$ & ${ }^{143} \mathrm{Nd} /{ }^{144} \mathrm{Nd}$ & $\varepsilon N d(t)$ & $\mathrm{T}_{\mathrm{DM}}$ & $\mathrm{T}_{2 \mathrm{DM}}$ \\
\hline PM20-10-Bb1 & 291.86 & 477.74 & 1.76774 & 0.709064 & 0.708806 & 5.79 & 39.32 & 0.088945549 & 0.512286 & -6.73 & 1057 & 1374 \\
\hline PM21-10-Bb1 & 221.19 & 604.07 & 1.05954 & 0.708442 & 0.708287 & 5.33 & 36.94 & 0.087177326 & 0.512288 & -6.68 & 1040 & 1371 \\
\hline PM24-1-Bb1 & 213.43 & 548.25 & 1.12645 & 0.708635 & 0.708471 & 5.80 & 40.38 & 0.086796746 & 0.512284 & -6.76 & 1042 & 1377 \\
\hline
\end{tabular}


The 29 analyzed areas in zircons used for $\mathrm{U}-\mathrm{Pb}$ dating (sample PM21-Bb5) were selected to carry out in-situ analyses for Lu-Hf isotope composition (test results are listed in Table 4). Among them, the ${ }^{176} \mathrm{Lu} /{ }^{177} \mathrm{Hf}$ ratios are all less than 0.002 , which indicates that ${ }^{176} \mathrm{Hf}$ accumulated little ${ }^{177} \mathrm{Hf}$ due to radiogenic decay during the geological evolution of magma sources. Therefore, the ${ }^{176} \mathrm{Hf} /{ }^{177} \mathrm{Hf}$ ratios measured can basically represent the Hf isotopic composition of zircon crystallization systems [68]. At the same time, the $f_{\mathrm{Lu} / \mathrm{Hf}}$ value of zircon ranges from -0.98 to -0.93 , with an average value of -0.97 , which is significantly smaller than that of silica-alumina (-0.72) [69] and silica-magnesium $(-0.34)$ [70]. Therefore, the $\mathrm{T}_{\mathrm{DM} 2}$ of zircon does not represent the age of tuff and its source rocks but only represents the age at which the source rock material from the depleted mantle or the average age of retention in the crust $[69,70]$. The zircon ${ }^{176} \mathrm{Hf} /{ }^{177} \mathrm{Hf}$ ratios of the samples are in the range of $0.282647-0.282816$; the corresponding $\varepsilon \mathrm{Hf}(t)$ values are widely distributed (-4.19-6.72), but most of them are located under the uniform chondrite reservoir; the zircon $\mathrm{Hf}$ isotopic one-stage model age $\mathrm{T}_{\mathrm{DM}}=0.42-0.85 \mathrm{Ga}$, and the two-stage age $\mathrm{T}_{\mathrm{DM} 2}=0.98-1.36 \mathrm{Ga}$.

\subsection{Crustal Thickness}

In the process of subduction and orogenic evolution, variation in crustal thickness is one of the important parameters affecting the geochemical characteristics of magmatic rocks [71-73]. The quantitative or semiquantitative estimation of crustal thickness is key to limiting deep geological processes and magmatic evolution. Chapman [74] and Profeta [75] stated that the $\mathrm{Sr} / \mathrm{Y}$ and $(\mathrm{La} / \mathrm{Yb})_{\mathrm{N}}$ of intermediate-acid magma have a linear relationship with crustal thickness on the basis of the comprehensive analysis of a large number of data. All the samples are applied to the empirical equation conditions, so the median samples (PM24-5-Bb1, PM21-6-Bb1) are selected to estimate the crustal thickness. (1) The relationships of thickness to the $\mathrm{Sr} / \mathrm{Y}$ value proposed by Chapman et al. (2015) [74], imply that, the crustal thickness of sample PM24-5-Bb1 is $68.5 \mathrm{~km}$ with an uncertainty of $7.2 \mathrm{~km}$, and that the crustal thickness of sample PM21-6-bb1 is $68.2 \mathrm{~km}$ with an uncertainty of $7.1 \mathrm{~km}$. (2) Applying the linear equation of thickness related to the $(\mathrm{La} / \mathrm{Yb})_{\mathrm{N}}$ value proposed by Profeta et al.(2015) [75], yields a crustal thickness derived from sample PM24$5-\mathrm{Bb} 1$ of $79.2 \mathrm{~km}$ with an uncertainty of $13.6 \mathrm{~km}$; and a crustal thickness derived from sample PM21-6-Bb1of $79.8 \mathrm{~km}$, with an uncertainty of $14.6 \mathrm{~km}$.

These results show that the Miocene Gangdese crustal thicknesses calculated by the values of $\mathrm{Sr} / \mathrm{Y}$ and $(\mathrm{La} / \mathrm{Yb})_{\mathrm{N}}$ are approximately $60-80 \mathrm{~km}$, which is a reasonable thickness and exhibits a state of substantial thickening. This result is consistent with the crustal thickness obtained by other independent geological methods in the study region [76,77]. 
Table 4. Zircon Hf isotopic data for check the Zongdangcun Formation.

\begin{tabular}{|c|c|c|c|c|c|c|c|c|c|c|c|c|c|c|}
\hline Sample & Age (Ga) & ${ }^{176} \mathrm{Yb} /{ }^{177} \mathrm{Hf}$ & $2 \sigma$ & ${ }^{176} \mathrm{Lu} /{ }^{177} \mathrm{Hf}$ & $2 \sigma$ & ${ }^{176} \mathrm{Hf} /{ }^{17} 7 \mathrm{Hf}$ & $2 \sigma$ & $\mathrm{T}_{\mathrm{DM}}(\mathrm{Ga})$ & $\mathrm{T}_{2 \mathrm{DM}}(\mathrm{Ga})$ & Hf(i) & $\varepsilon \mathbf{H f}(\mathbf{0})$ & $\varepsilon \mathbf{H f}(\mathbf{t})$ & $2 \sigma$ & $f_{\mathrm{Lu} / \mathrm{Hf}}$ \\
\hline PM21-JD1-1 & 0.0105 & 0.033373 & 0.000867 & 0.001171 & 0.000028 & 0.282792 & 0.000023 & 0.66 & 1.04 & 0.282792 & 0.70 & 0.92 & 0.81 & -0.96 \\
\hline PM21-JD1-2 & 0.0105 & 0.018638 & 0.000056 & 0.000619 & 0.000007 & 0.282800 & 0.000021 & 0.63 & 1.02 & 0.282800 & 0.98 & 1.21 & 0.75 & -0.98 \\
\hline PM21-JD1-4 & 0.0104 & 0.021760 & 0.000485 & 0.000682 & 0.000013 & 0.282782 & 0.000021 & 0.66 & 1.06 & 0.282782 & 0.36 & 0.58 & 0.73 & -0.98 \\
\hline PM21-JD1-5 & 0.0102 & 0.018659 & 0.000048 & 0.000647 & 0.000002 & 0.282796 & 0.000018 & 0.64 & 1.03 & 0.282796 & 0.86 & 1.08 & 0.64 & -0.98 \\
\hline PM21-JD1-6 & 0.0104 & 0.046085 & 0.001602 & 0.001391 & 0.000061 & 0.282657 & 0.000028 & 0.85 & 1.34 & 0.282657 & -4.07 & -3.85 & 1.01 & -0.96 \\
\hline PM21-JD1-7 & 0.0104 & 0.045220 & 0.000237 & 0.001276 & 0.000006 & 0.282743 & 0.000023 & 0.73 & 1.15 & 0.282742 & -1.04 & -0.82 & 0.80 & -0.96 \\
\hline PM21-JD1-9 & 0.0105 & 0.024186 & 0.000112 & 0.000671 & 0.000002 & 0.282647 & 0.000022 & 0.85 & 1.36 & 0.282647 & -4.41 & -4.19 & 0.78 & -0.98 \\
\hline PM21-JD1-13 & 0.0102 & 0.012335 & 0.000181 & 0.000382 & 0.000005 & 0.282800 & 0.000020 & 0.63 & 1.02 & 0.282800 & 0.99 & 1.21 & 0.69 & -0.99 \\
\hline PM21-JD1-14 & 0.0102 & 0.023904 & 0.000677 & 0.000654 & 0.000017 & 0.282753 & 0.000025 & 0.70 & 1.12 & 0.282753 & -0.67 & -0.46 & 0.88 & -0.98 \\
\hline PM21-JD1-15 & 0.0103 & 0.083510 & 0.000518 & 0.002139 & 0.000012 & 0.282764 & 0.000028 & 0.71 & 1.10 & 0.282763 & -0.30 & -0.09 & 1.00 & -0.94 \\
\hline PM21-JD1-16 & 0.0102 & 0.031547 & 0.000490 & 0.000937 & 0.000014 & 0.282736 & 0.000025 & 0.73 & 1.16 & 0.282735 & -1.29 & -1.07 & 0.87 & -0.97 \\
\hline PM21-JD1-17 & 0.0104 & 0.029805 & 0.000490 & 0.001050 & 0.000020 & 0.282813 & 0.000027 & 0.62 & 0.99 & 0.282813 & 1.45 & 1.67 & 0.96 & -0.97 \\
\hline PM21-JD1-18 & 0.0102 & 0.030783 & 0.000788 & 0.000999 & 0.000029 & 0.282793 & 0.000023 & 0.65 & 1.03 & 0.282793 & 0.74 & 0.96 & 0.81 & -0.97 \\
\hline PM21-JD1-19 & 0.0102 & 0.025492 & 0.000234 & 0.000976 & 0.000008 & 0.282816 & 0.000038 & 0.62 & 0.98 & 0.282815 & 1.54 & 1.76 & 1.36 & -0.97 \\
\hline PM21-JD1-20 & 0.0104 & 0.016714 & 0.000087 & 0.000545 & 0.000010 & 0.282756 & 0.000027 & 0.69 & 1.12 & 0.282756 & -0.55 & -0.33 & 0.96 & -0.98 \\
\hline PM21-JD1-21 & 0.0100 & 0.026894 & 0.000162 & 0.001030 & 0.000008 & 0.282729 & 0.000021 & 0.74 & 1.18 & 0.282728 & -1.53 & -1.32 & 0.73 & -0.97 \\
\hline PM21-JD1-23 & 0.0120 & 0.029601 & 0.000749 & 0.000930 & 0.000026 & 0.282699 & 0.000022 & 0.78 & 1.24 & 0.282699 & -2.58 & -2.32 & 0.76 & -0.97 \\
\hline PM21-JD1-24 & 0.0100 & 0.040209 & 0.000458 & 0.001382 & 0.000016 & 0.282774 & 0.000021 & 0.68 & 1.08 & 0.282773 & 0.06 & 0.27 & 0.74 & -0.96 \\
\hline PM21-JD1-26 & 0.0098 & 0.036028 & 0.000528 & 0.001025 & 0.000007 & 0.282774 & 0.000024 & 0.68 & 1.08 & 0.282774 & 0.07 & 0.28 & 0.86 & -0.97 \\
\hline PM21-JD1-28 & 0.0100 & 0.027640 & 0.000600 & 0.000701 & 0.000013 & 0.282776 & 0.000026 & 0.67 & 1.07 & 0.282776 & 0.15 & 0.36 & 0.90 & -0.98 \\
\hline PM21-JD1-30 & 0.0106 & 0.020169 & 0.000440 & 0.000791 & 0.000015 & 0.282790 & 0.000025 & 0.65 & 1.04 & 0.282790 & 0.63 & 0.86 & 0.89 & -0.98 \\
\hline PM21-JD1-33 & 0.0100 & 0.070652 & 0.002432 & 0.002179 & 0.000048 & 0.282791 & 0.000025 & 0.67 & 1.04 & 0.282790 & 0.66 & 0.87 & 0.87 & -0.93 \\
\hline PM21-JD1-35 & 0.0108 & 0.019444 & 0.000324 & 0.000511 & 0.000007 & 0.282739 & 0.000020 & 0.72 & 1.16 & 0.282739 & -1.18 & -0.95 & 0.70 & -0.98 \\
\hline PM21-JD1-37 & 0.0106 & 0.056193 & 0.001160 & 0.001349 & 0.000025 & 0.282956 & 0.000027 & 0.42 & 0.66 & 0.282956 & 6.50 & 6.72 & 0.95 & -0.96 \\
\hline PM21-JD1-38 & 0.0102 & 0.038387 & 0.000275 & 0.001250 & 0.000011 & 0.282794 & 0.000025 & 0.65 & 1.03 & 0.282794 & 0.78 & 1.00 & 0.88 & -0.96 \\
\hline PM21-JD1-39 & 0.0100 & 0.064006 & 0.000778 & 0.001629 & 0.000015 & 0.282791 & 0.000021 & 0.66 & 1.04 & 0.282791 & 0.67 & 0.88 & 0.73 & -0.95 \\
\hline PM21-JD1-40 & 0.0102 & 0.020273 & 0.000642 & 0.000561 & 0.000015 & 0.282699 & 0.000020 & 0.77 & 1.24 & 0.282699 & -2.57 & -2.35 & 0.71 & -0.98 \\
\hline
\end{tabular}




\section{Discussion}

The major and trace elements, bulk rock $\mathrm{Sr}-\mathrm{Nd}$ isotopes, zircon $\mathrm{U}-\mathrm{Pb}$ geochronologic and zircon Hf isotopes characteristics of the adakitic rocks in Tibet place important constraints on the geodynamic evolution of southern Tibet after the collision of the Indian and Asian plates. The crystal-vitreous tuffs do not have rock fragments, indicating that there is no heterogeneous material derived from crustal rocks. In addition, the samples have the same REE normalized patterns and a similar Sr-Nd composition, which suggests that the crystal-vitreous tuff preserves the composition of the felsic magmas.

\subsection{Origin and Petrogenesis of The Zongdangcun Group Adakitic Rock}

The adakitic rocks of the Zongdangcun Formation in the Wuyu Basin are characterized by high $\mathrm{Sr}$, low $\mathrm{Y}$, high $\mathrm{Sr} / \mathrm{Y}$, and $\mathrm{La} / \mathrm{Yb}$ ratios and fall into the adakitic rock field (Figure 7). Due to the occurrence of $\mathrm{Cr}, \mathrm{Sc}$, and $\mathrm{Y}$ and HREEs in garnet, these studied samples show that the content of the above elements is low and that the Sc content is almost stable with changes in the $\mathrm{Cr}$ content, indicating that there are a lot of garnet residues in the source area and the samples were formed in a deeper depth. Moreover, the relative depletion of $\mathrm{Ba}$ with respect to $\mathrm{Rb}$ in the primitive, incompatible mantle-normalized element patterns (Figure 6B) indicates that amphibole might also be in a residual phase, reflecting the partition coefficient of $\mathrm{Ba}$ is higher than that of $\mathrm{Rb}[78,79]$.

Although the partial melting of subducted oceanic crust [69] is the former model for the formation of adakitic rocks, the adakitic rocks of the Zongdangcun Formation were formed in the Miocene (Table 1), and the Gangdese batholith was in the post-collisional stage $[12-14,44,46,50,80,81]$. The subduction of the Neotethys Oceanic crust ended, and the lithospheric subduction of the Neotethys Oceanic crust broke off during the period of $\sim 50 \mathrm{Ma}$ [82-84]. The geochemical characteristics of the samples, such as low $\mathrm{MgO}$, $\mathrm{Ni}$, and $\mathrm{Cr}$ concentration, are significantly different from those of adakites formed by subduction slab melting (Figure 8). It can be ruled out that this mechanism is the origin of Miocene adakites in southern Tibet. This paper will focus on the following four possible genetic mechanisms: (1) melting of mantle peridotite under water-bearing conditions [21,50]; (2) fractional crystallization and assimilation of basalt melt under high or low pressure [85-87]; (3) partial melting of subducted continental crust/delamination of the crust [13]; and (4) partial melting of the thickened lower crust in a post-collisional environment $[12,13,87-91]$.

The low-degree partial melting of mantle peridotite only produces andesitic melt and cannot directly form a large amount of felsic melt, while the high-degree separation crystallization model of basaltic magma requires a larger supply of basaltic parent magma [92]. During the post-collisional period, there were only a small amount of mafic magmatic rocks in the western Gangdese belt $[44-46,93,94]$. However, the magmatic rocks of the Zongdangcun Formation exposed in the Wuyu Basin and its periphery are mainly rhyolite volcanic rocks, and the area lacks a large amount of contemporary mafic magmatic rocks $[3,40,47]$. In addition, Figure $8 \mathrm{~B}, \mathrm{E}$ show that the formation of adakitic magmas in the Zongdangcun Formation is not directly related to fractional crystallization, but mainly due to partial melting of the lower crust. Therefore, we propose that the melting of the mantle peridotite and the fractional crystallization of basaltic melts are not ideal models for the formation of adakitic rocks in the Zongdangcun Formation. 

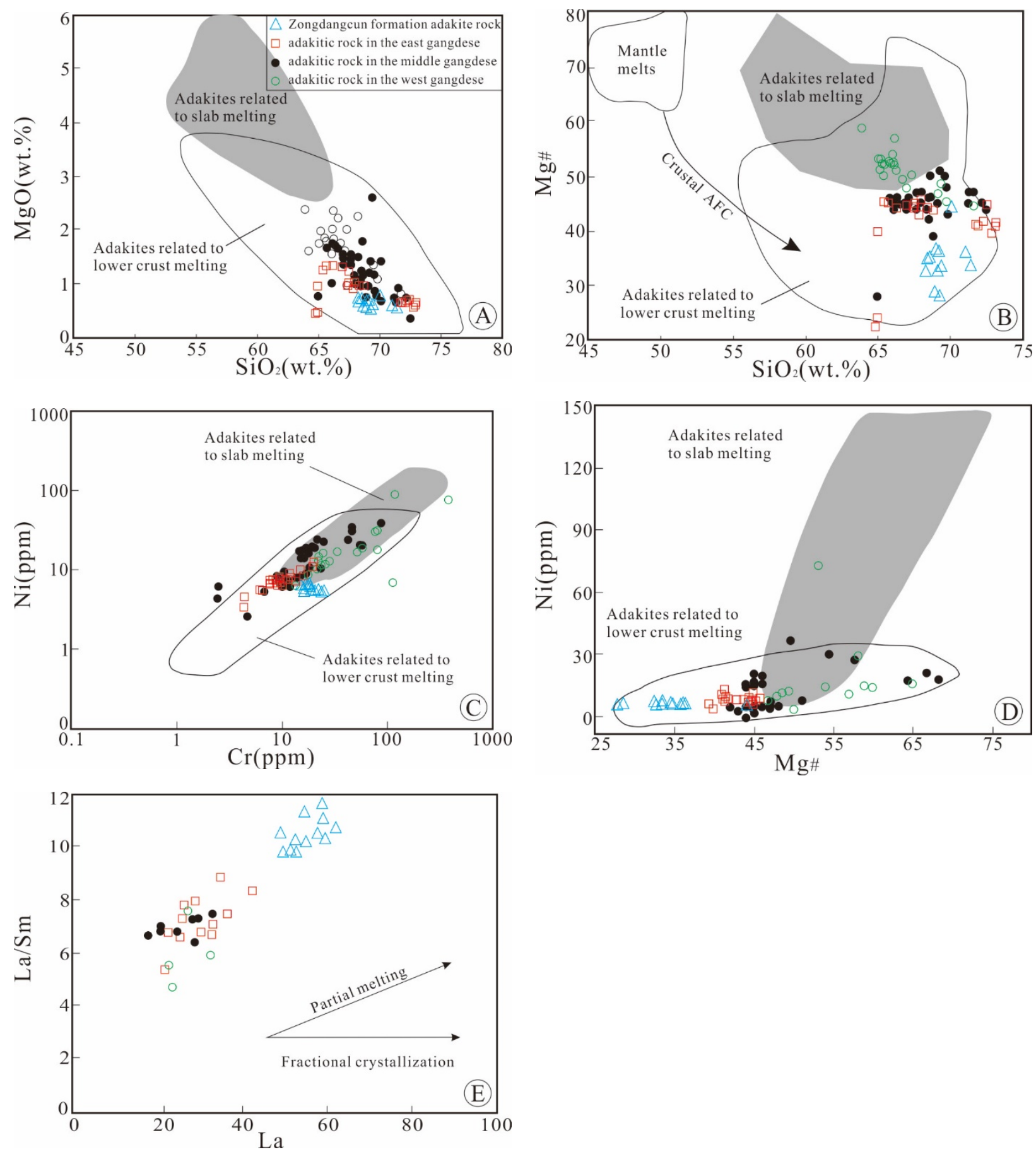

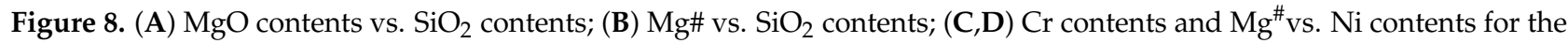
Miocene adakitic rocks from the Lhasa terrane, and (E) La vs. La/Sm diagrams. Mantle AFC curves are after [95]. The field of adakites related to slab melting and lower crustal melting is from $[43,96]$. Data come from the same source as Figure 4.

The Eocene-Miocene leucogranites in the Tethyan Himalayan orogen are believed to have been formed by the melting of the continental crust of India, with similarly high ${ }^{87} \mathrm{Sr} /{ }^{86} \mathrm{Sr}$ and low ${ }^{143} \mathrm{Nd} /{ }^{144} \mathrm{Nd}$ values and low zircon $\mathrm{Hf}$ isotope compositions [97-99]. The adakitic rocks of the Zongdangcun Formation have higher $\varepsilon \mathrm{Hf}(\mathrm{t})(-4.19-6.72)$ and $\varepsilon \mathrm{Nd}(\mathrm{t})(-6.76--6.68)$, which are quite different from the melts from Indian continental crust. In addition, the melt derived from the partial melting of subducted or delaminated continental crust will interact with the mantle peridotite during the ascending process, which makes it rich in magnesium $\left(\mathrm{MgO}>1.5 \%, \mathrm{Mg}^{\#}>50\right)$, relatively low in silicon $\left(\mathrm{SiO}_{2}=56-68 \%\right)$ 
and high in compatible elements ( $\mathrm{Cr}$ and Ni), [36,61,70,88,90,91,100-102], which is not consistent with the geochemical characteristics of the studied samples $\left(\mathrm{SiO}_{2}=68.27-71.37 \%\right.$, $\mathrm{MgO}=0.50-0.69 \%, \mathrm{Mg} \#=27.71-43.93, \mathrm{Cr}=5.22-24.71$, and $\mathrm{Ni}=5.33-6.49)$. Furthermore, the geophysical data indicates that a large volume of continental lithospheric mantle and the lower crust of the Lhasa block are still preserved below the Lhasa block [77]. Thereby excluding the possibility of being formed in the subducted or delaminated lower continental crust.

Herein, we propose that the main body of adakitic rocks of the Zongdangcun Formation in the Wuyu Basin is derived from the partial melting of the thickened lower crust. The amount of plagioclase crystals in the samples is small, and there is a slight negative Eu anomaly. The evidence shows that these adakites didn't experience extensive plagioclase accumulation in crustal magmatic reservoirs. The adakitic rocks of the Zongdangcun Formation are peraluminous, indicating that there is a large volume of Al-rich crustal components in the magmatic source [42], and the whole-rock geochemical characteristics (low $\mathrm{MgO}, \mathrm{Cr}, \mathrm{Ni}$, and high $\mathrm{K}_{2} \mathrm{O}$ contents) of the samples support our view. On the Time-Hf(t) diagram, the sample zircon $\varepsilon \mathrm{Hf}(\mathrm{t})$ has a wide distribution range and is mainly negative, mostly falling below the $\mathrm{T}_{\mathrm{DMC}}=1.0 \mathrm{Ga}$ line (Figure 9), and the $\mathrm{T}_{\mathrm{DM} 2}$ of $\mathrm{Hf}$ is relatively old at 0.98-0.1.36 Ga (Figure 9), which different from Indian crust $(\varepsilon \mathrm{Hf}(\mathrm{t})<-8)$ [5] and Lhasa terrane $(\varepsilon \mathrm{Hf}(\mathrm{t})=-3--14)$ and Gangdese batholith $(\varepsilon \mathrm{Hf}(\mathrm{t})$ is usually positive) [42]. This result reflects that the magma has the characteristics of a multip source. In addition, it shows that the adakitic magmas may be dominated by thickening crust and remelting on the southern margin of the Gangdese block. The Sr-Nd isotopic composition of the sample is obviously different from that of adakitic rock formed by partial melting of the subduction crust of India [15,103], and it is similar to adakitic rocks and volcanic rocks of the Linzizong Group formed by partial melting of the thickened lower crust of the Gangdese belt during the Oligocene-Miocene (Figure 9). The latter is also considered to be formed by partial melting of the thickened mafic lower crust [41,104]. However, the Sr-Nd isotopic composition of the sample is also different from that of the adakitic rocks formed by partial melting of the thickened crust during the Oligocene-Miocene in the Gangdese zone and the volcanic rocks of the Linzizong Group. The higher $\left({ }^{87} \mathrm{Sr} /{ }^{86} \mathrm{Sr}\right)_{\mathrm{i}}$ and lower $\varepsilon \mathrm{Nd}(\mathrm{t})$ indicates that enriched components from ancient crust are involved in the magma genesis process. The geochemical characteristics of Sr-Nd isotopes also indicate that the source of magma does not simply originate from the partial melting of the thickened crust. The main source of its enriched composition may be the ancient basement of Lhasa at the bottom of the thickened crust of the Indian subducted continental crust at the bottom of the thickened crust. The samples in Figure 10 plot on the mixed two-terminal element curve of the Cenozoic thickened crust and the leucogranite of the Himalayan orogenic belt representing the isotope composition of the Indian continental crust [98], which is quite different from the isotope composition of the Lhasa lower crust [45]. Therefore, we consider the subducting Indian ancient continental crust to be the most probable source to provide enriched components for adakitic magmas. In addition, from the analysis of the distribution position of magmatic rocks, the pre-Cambrian crystalline basement reported at the southern margin of the Lhasa block is mainly distributed in the eastern Himalayan tectonic node, but the Wuyu Basin is located in the middle section of the southern margin of the southern Lhasa block, so the ancient basement materials distributed in the eastern tectonic node cannot participate in the formation of rocks. At the same time, taking into account the impact of crustal shrinkage caused by the collision between India and Eurasia since the Cenozoic, the Wuyu Basin exhibits a long geographic distance from the central Lhasa terrane during the Miocene, resulting in the ancient basement in the middle of Lhasa block is difficult to participate in the genesis of adakitic rocks of Zongdangcun Formation. It is also difficult to establish the basement participation in the magma genesis of adakitic rocks in the Zongdangcun Formation. Therefore, we thought that the main reason for the high $\left({ }^{87} \mathrm{Sr} /{ }^{86} \mathrm{Sr}\right)_{\mathrm{i}}$ and low $\varepsilon \mathrm{Nd}(\mathrm{t})$ and $\varepsilon \mathrm{Hf}(\mathrm{t})$ characteristics (Figures 9 and 10) of the 
Zongdangcun Formation adakitic rocks relative to the adakitic rocks of the Linzizong Group is the addition of enriched ancient Indian crustal components [15,16,42,97,105-108].

On the basis of the linear relationship of the $\mathrm{Sr} / \mathrm{Y}$ value, $(\mathrm{La} / \mathrm{Yb})_{\mathrm{N}}$ value and magma formation depth [76,77], the result of the depth of magma formation (Figure 11) is close to the crustal thickness of the southern Tibetan Plateau, which is approximately $74 \mathrm{~km}$ [109] and further supports that it originated from the crust-mantle boundary at the bottom of the thickened crust of the Qinghai-Tibet Plateau.

As the lithosphere of the Qinghai-Tibet Plateau during the continental collision and subduction process, due to the shortening of the Cenozoic and the uniform thickening of the crust, resulted in gravitational instability, the upwelling of hot asthenospheric material heated the lithosphere mantle and triggered its partial melting to form the potassium-rich magmas. The K-rich mafic magma migrated to the surface or remained in the subsurface along the E-W extensional tectonic control [47]. Potassium-rich magma trapped in the lower crust heated the surrounding mafic lower crust, promoting the partial melting of the crust to form adakitic magma. As the amount of fluid/melt released by subducting continental crust is much lower than that of the oceanic crust subduction zone, the intensity of magmatism above the continental subduction zone is obviously lower than that of the oceanic continental subduction zone [110]. Compared with the large amount of granite in the Linzizong group formed during the subduction period of the Neotethyan oceanic crust in the Gangdise belt $[41,104]$, the area of the adakite in Miocene is very small, which is consistent with the characteristics of the weak magmatism induced by subduction of the continental plate. Therefore, the incorporation of fluids/melts from the subducting Indian plates and the decompression of extensional structures may be the main contributing processes for the formation of adakitic parent magma.

Herein, we propose that the post-collisional adakitic magmatic rocks in the middle segment of the southern margin of the Gangdese belt are mainly derived from partial melting of the thickened lower crust (mainly the lower crust of the Lhasa block with the addition of minor ancient Indian continental crust), with garnet and amphibole remaining in the source area. The upwelling of the asthenosphere provided the heat, while the addition of fluid/melt and the decompression of mafic lower crust triggered the partial melting of the mafic lower crust in the east-west extensional setting.

\subsection{Source Region for The Miocene Adakitic Rocks}

During the northward subduction of the Indian continent during Oligocene to Miocene, magmatic rocks consist of ultrapotassic rocks, potassic intermediated acid rocks and adakitic rocks are developed in the Lhasa block in southern Tibet $[3,45,97]$. Among them, the ultrapotassic rocks are mainly distributed in the middle of the Lhasa block and the area to the west of $87^{\circ} \mathrm{E}$ in a discontinuous band, and the magmatic activity timeline shows a trend of decreasing ages from west to the east $[47,97]$. The post-collisional adakitic rocks in the Gangdese belt were mainly formed between $33 \mathrm{Ma}$ and $8 \mathrm{Ma}$ [4] and distributed along the southern margin of the Gangdese belt overall. They mainly occur in the eastern segment of the structural belt and sporadically occur in the middle and western segments [3,12-14,16,48,61,97,105,111-114]. The adakitic rocks in the eastern and western parts of the Gangdese belt have geochemical characteristics similar to those of the samples produced in the middle of the tectonic belt (Figures 5 and 7), so we believe they may have similar sources of magma. However, the geochemical composition of the Miocene adakitic rocks in southern Tibet has the characteristics of regular changes from west to the east along the structural belt (Figures 9 and 10). According to the change of geochemical characteristics, the structural belt is divided into eastern, middle, and western sections along the $84^{\circ} \mathrm{E}$ and $90^{\circ} \mathrm{E}$ lines. 

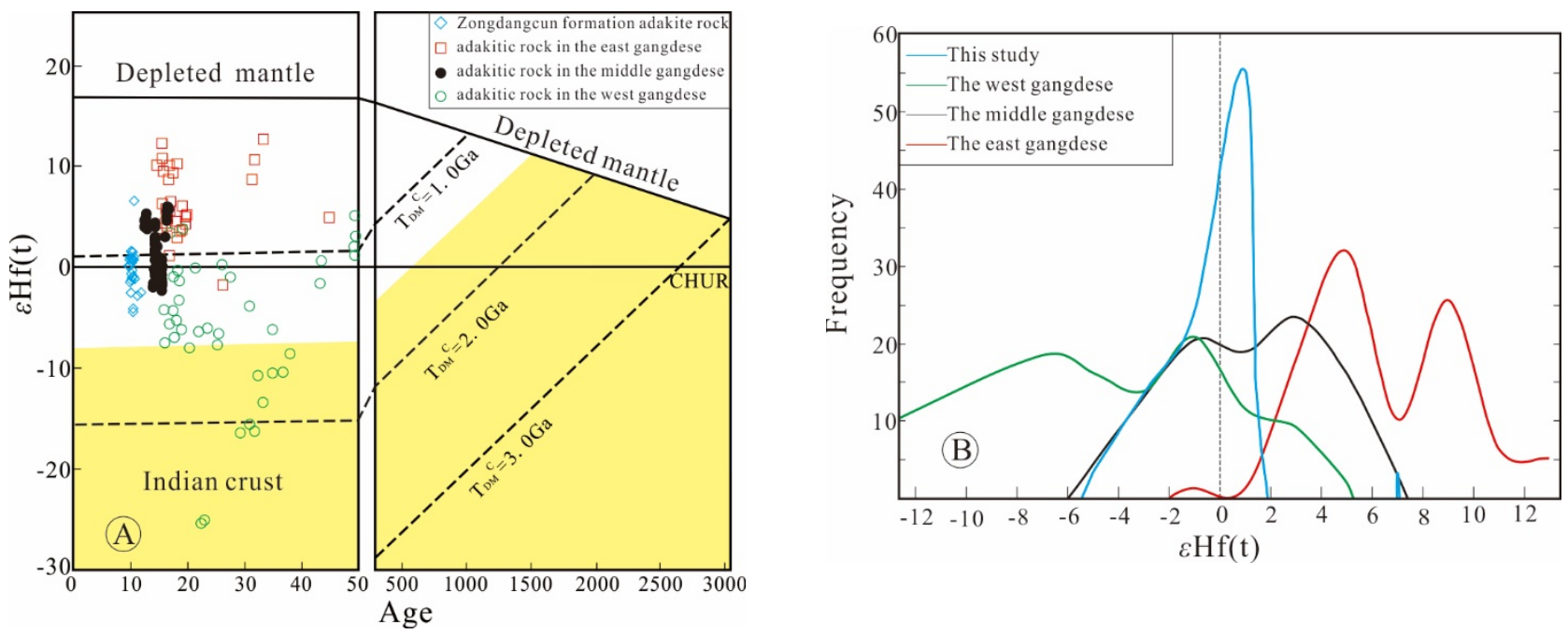

Figure 9. (A) Zircon Hf isotopic characteristics of Gangdese batholith granite; (B) The frequency of $\varepsilon \mathrm{Hf}(\mathrm{t})$ values of the Miocene adakitic rock in the esttern and middle and western of the Gangdese belt. Plots of $\varepsilon \mathrm{Hf}(\mathrm{t})$ values vs. Ages of zircons from the Miocene adakitic rock in the Gangdese. Data sources: The Miocene adakitic rock data come from [15,49,61,108,115]; the yellow area denotes the evolution field of the Indian continental crust [5].

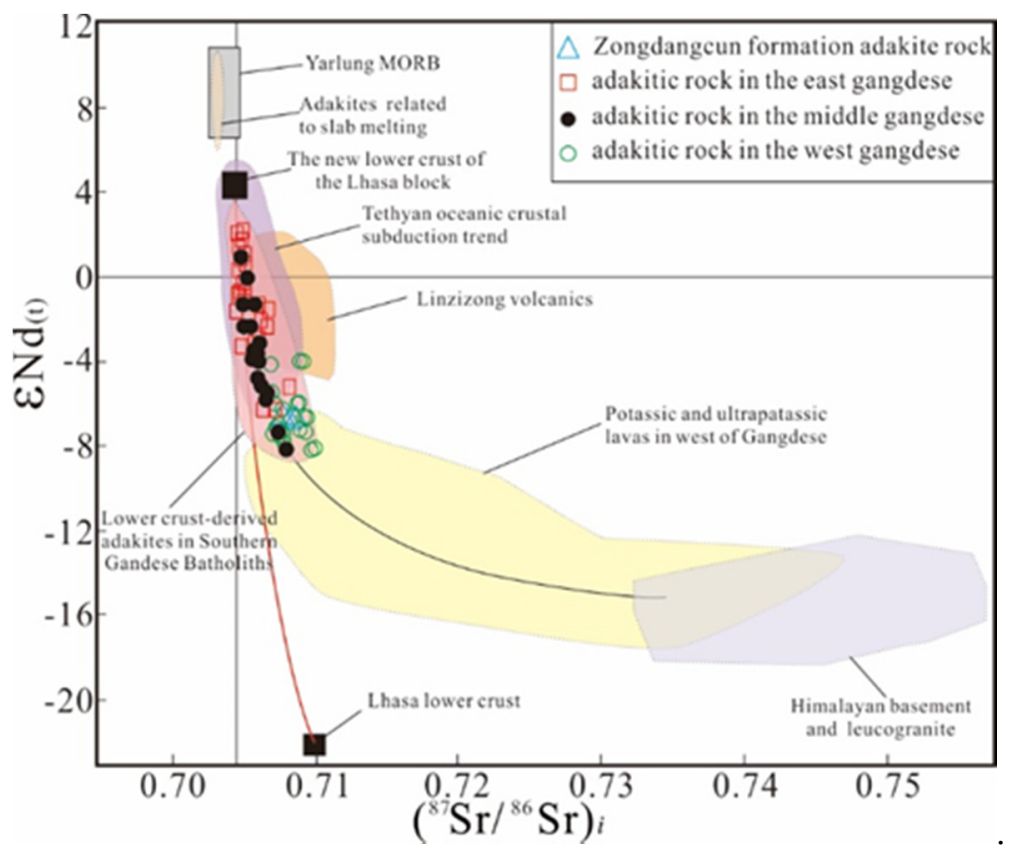

Figure 10. $\left({ }^{87} \mathrm{Sr} /{ }^{86} \mathrm{Sr}\right) \mathrm{i}-\varepsilon \mathrm{Nd}(\mathrm{t})$ diagram of Gangdese. Nd-Sr isotopic compositions of the Miocene adakitic rocks in the Lhasa terrane. The Miocene adakitic rocks from [13-15,21,50]. The Yarlung MORB from [11], the new crust of the Lhasa block ( as Dazi basalt) from [98]; lower crust from [45]; the ancient Indian crust (as leucogranite of the Himalayan ) form [107]. Data come from the same source as Figure 4.

The adakitic rocks in the eastern section of the southern margin of the Gangdese belt are mostly produced by ore-bearing porphyries, which have become an important part of the Gangdese porphyry copper belt [13]. The zircon Hf isotopes of the postcollisional adakitic magma in the eastern section of the Gangdese magmatic belt show depletion characteristics, with higher $\varepsilon \mathrm{Hf}(\mathrm{t})$ values. Some Hf isotopic compositions show enriched composition (Figure 9), suggesting that the main body of adakitic magma in the eastern Gangdese belt came from the depleted source and that a small amount of enriched components were involved. The Miocene adakitic rocks in the western section of the 
Gangdese belt are mostly calc-alkaline volcanic rocks [12], with minor (ultra-) potassic volcanic rocks. These adakitic rocks have geochemical characteristics and magmatic zircon $\mathrm{Hf}$ isotope model ages similar to those of eastern adakitic rocks, indicating that the main body of Miocene adakitic magma in the eastern and western Gangdese belts may have a similar source. However, the $\varepsilon \mathrm{Hf}(\mathrm{t})$ value of the magmatic zircon from the western Miocene adakitic rock is lower than those in the eastern section with mostly negative and a large distribution range, indicating that the western Miocene adakitic rock may derive from a much older continental crust or much less contribution of mantle materials. The $\mathrm{Hf}$ isotopic composition range of adakites in the middle section of the Gangdese belt is between the $\varepsilon \mathrm{Hf}(\mathrm{t})$ values of adakitic rocks in the eastern and western sections of the same period. Zircon $\varepsilon \mathrm{Hf}(\mathrm{t})$ value of these Miocene adakitic rocks increases substantially from west to east, suggesting that the decreased contribution of the ancient Indian crust during the formation of the thickened lower crust (Figure 9A,B).

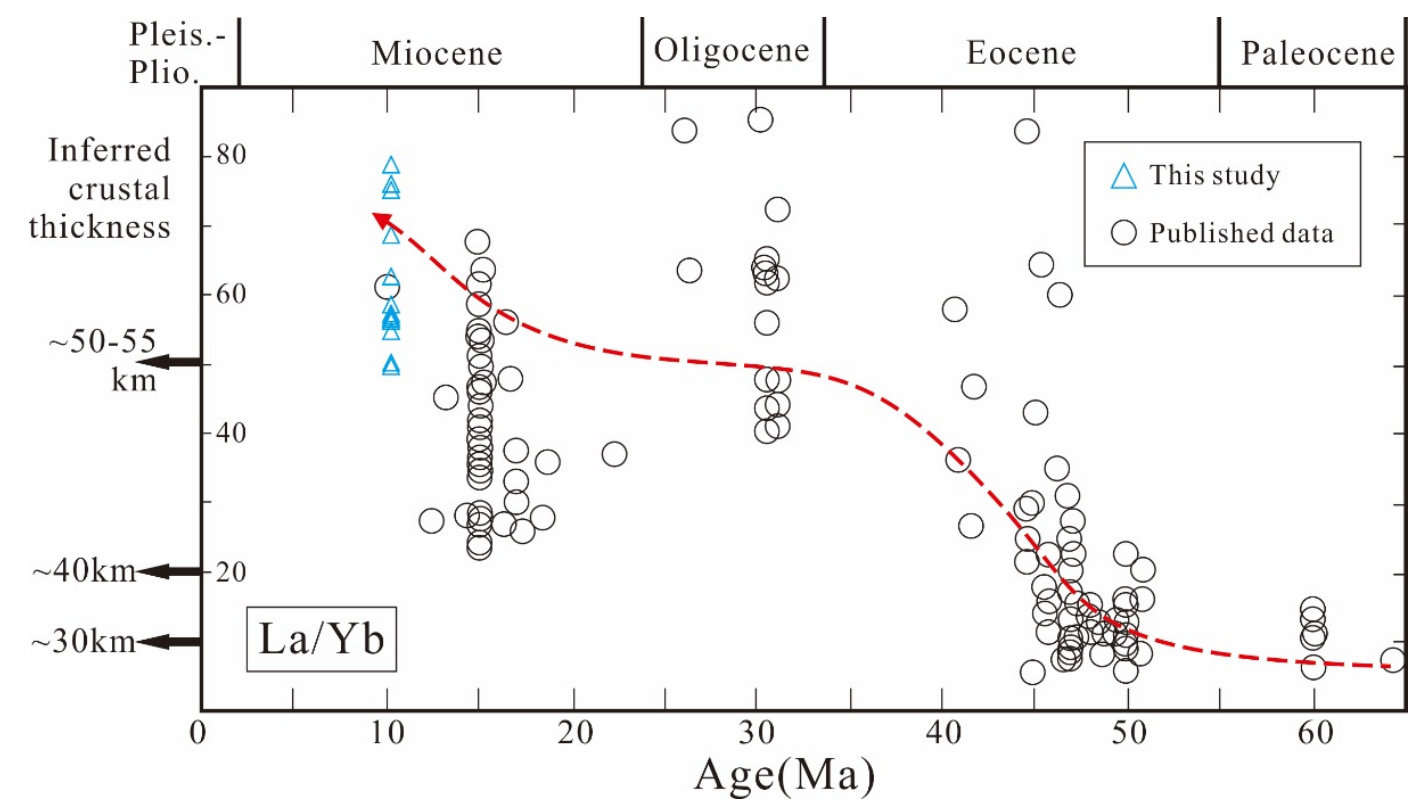

Figure 11. Plots of $\mathrm{La} / \mathrm{Yb}$ ratios vs. magmatic ages for the post-collisional adakites in southern Tibet. Data come from the same source as Figure 4.

The $\varepsilon \mathrm{Nd}(\mathrm{t})-\left({ }^{87} \mathrm{Sr} /{ }^{86} \mathrm{Sr}\right)_{\mathrm{i}}$ diagram of the adakitic rocks in the Gangdese belt clearly reflect the changing trend of the material contribution of the ancient crust. The Sr-Nd isotope composition of Miocene adakitic magmatic rocks in the Gangdese belt is distributed in both directions of more depletion and more enrichment (Figure 10). This reflects that the magma source or during magma evolution, there may be both the contribution of depleted mantle-derived materials and the involvement of ancient crustal enriched components. The Sr-Nd isotopes of Miocene adakitic rocks in southern Tibet all plot on or near the mixing line between the juvenile Lhasa thickened lower crust and the ancient Indian crust (represented by the leucogranite of the Himalayas). The adakitic rocks in the eastern section of the southern margin of the Gangdese belt have the characteristics of low $\left({ }^{87} \mathrm{Sr} /{ }^{86} \mathrm{Sr}\right)_{i}$ and high $\varepsilon \mathrm{Nd}(\mathrm{t})$ signaling depleted source areas, while the source areas of the adakitic rocks in the western section are more enriched. The $\mathrm{Sr}-\mathrm{Nd}$ isotope variation range in the middle section of the study area is widely distributed, showing obvious transition characteristics. Sr-Nd isotope analysis shows that $\mathrm{Hf}$ isotope analysis is consistent, which strongly indicates that there are key source differences among the three eastern, central and western sections of Gangdese. The eastern section has a lesser contribution from the Indian continental crust, compared to the western section. The large-scale development of porphyry copper deposits in the eastern part of the contemporaneous structure may also 
be related to the fact that the magma in the eastern part of the structure originated from mantle source material [116].

In summary, the post-collisional adakitic magma generation along the southern margin of the Gangdese belt has a similar origin. The main body of adakitic magma is formed by the partial melting of thickened crust, and the difference in quantity involved in the ancient crust of India may be the main reason for the geochemical heterogeneity of adakitic magma after the collision.

\subsection{Tectonic Implication}

The geodynamic implications of Miocene adakitic magmatism on the Tibetan Plateau are closely related to the post-collisional interactions between the Eurasian and Indian plates. Previous studies have shown that the thickness of the Tibetan Plateau is approximately twice that of the average continental crust thickness $(-70 \mathrm{~km})[117,118]$. Most geoscientists hypothesize that the formation of the thick crust of the Qinghai-Tibet Plateau is mainly related to the compression and shortening of the crust caused by the Cenozoic collision between India and Eurasia. The upwelling of mantle material on the QinghaiTibet Plateau and the underplating of magma also made an important contribution to the thickening of the crust $[12,13,15,21,40,50]$. According to the linear relationship between the $\mathrm{Sr} / \mathrm{Y}$ value and the $(\mathrm{La} / \mathrm{Yb})_{\mathrm{N}}$ value $[74,75]$. The depth of magma formation depth is estimated, and the results are shown in Figure 11. The crust of the Gangdese belt in southern Tibet has thickened rapidly since 40 Ma [12,108,112,119], which is consistent with the collision between the Indian plate and the Asian plate as previous have shown. Therefore, we assume that the crust of the Qinghai-Tibet Plateau is uniformly shortened and thickened due to the collision of the Indian-Asian continent.

At present, the more meaningful dynamic models for simulating post-collisional magmatism in the Gangdese $[44,93,108]$ mainly include lithospheric mantle convective thinning [44,93] and Indian plate rotation/disconnection [116,120]. In this period, there is a lack of massive basic magmatism in the study area $[3,37,45,47]$, so the mantle plume model is usually not used as the tectonic origin of post-collisional magmatism in the Gangdese belt. The lithospheric convective thinning model emphasizes that the lithospheric mantle is gravitationally unstable due to the shortening of the Cenozoic crust and uniform thickening. Part of the continental lithospheric mantle is replaced by the upwelling asthenosphere, which provides heat for the partial melting of the lithospheric mantle and lower crust and is accompanied by surface uplift. The Indian plate rotation/detachment model suggests that plate rotation/detachment caused the upwelling of asthenospheric material in the lower part of the Indian lithosphere, which led to the melting of the lithospheric mantle in the lower part of the Tibetan Plateau. However, it is difficult to explain both the N-S rift system caused by the E-W extension and the E-W distribution of the post-collisional magmatic rocks in the Gangdese belt [50]. Integrating post-collisional magmatism and its relationship with the north-south rift system [121], combined with the geophysical data of the east-west subduction angle of the Indian subducting plate in the Gangdese zone [24], a model simulating the tearing and segmented subduction of the Indian plate front is proposed (Figure 12).

The composition of the Gangdese granite in southern Tibet also suggests that the magma source is mainly juvenile Lhasa thicked lower crust [112,113,122]. The Indian plate subducted toward the Lhasa block at a high angle in the eastern part of the Gangdese belt [24], which provided the environment for the upwelling of high-temperature asthenosphere to contact the Cenozoic lower crust. The Sr-Nd isotopes of adakitic rocks and the Hf isotope characteristics of magmatic zircons from the post-collision activity in the eastern section of the Gangdese magma belt indicate that the magma originated from a depleted source area (Figures 9 and 10), suggesting that igneous rocks were sourced from a mafic lower crust in the thickened orogeny. Individual samples with enrichment characteristics indicate that a small number of ancient Indian crustal components are involved in the eastern part of the Gangdese belt. The Sr-Nd isotopes of the adakitic rocks in the western 
section of the Gangdese belt and the Hf isotopic composition of magmatic zircon show that the source magma in the Western Gangdese belt is more enriched than that in the eastern Gangdese belt, suggesting that the participation ratio of Indian ancient crust end members in the post-collisional adakitic rocks in the western Gangdese belt is higher than that in the eastern Gangdese belt. Seismic data show that the Indian plate subducted at a low angle in the western segment of the collision zone [24]. Predecessors have performed much research here and ruled out the possibility that large-scale lithospheric delamination, slab separation, or a sudden increase in the subduction angle of the Indian continental slab occurred in the western section of the Gangdese belt, which then created a large amount of asthenospheric material $[3,12,46,81,83,120]$. As the subduction angle of the Indian plate in the western part of the Gangdese belt is gentle, the amount of asthenospheric material that can upwell through the tearing window of the subduction plate in the western part of the Gangdese belt under such geological conditions is limited, and cannot act as a major heat source and depleted material for the thickened lower crust. Consistent with the isotope ratios, the involvement of ancient Indian crust during the formation of Miocene adakitic magma in the western part of the Gangdese belt constitutes a higher contribution. The Miocene adakitic magmas in the middle part of the Gangdese belt are located between the two and have obvious transitional geochemical characteristics. In this case, the characteristics of the Sr-Nd isotope and magmatic zircon Hf isotope of the post-collision formation in the Gangdese belt show that the source magma is enriched in the western part of the structural belt and gradually depleted to the east, which can be considered strong evidence that the material contribution of the Indian subducted continental crust in the Gangdese structural belt gradually decreases during the post-collisional adakitic magmatism.
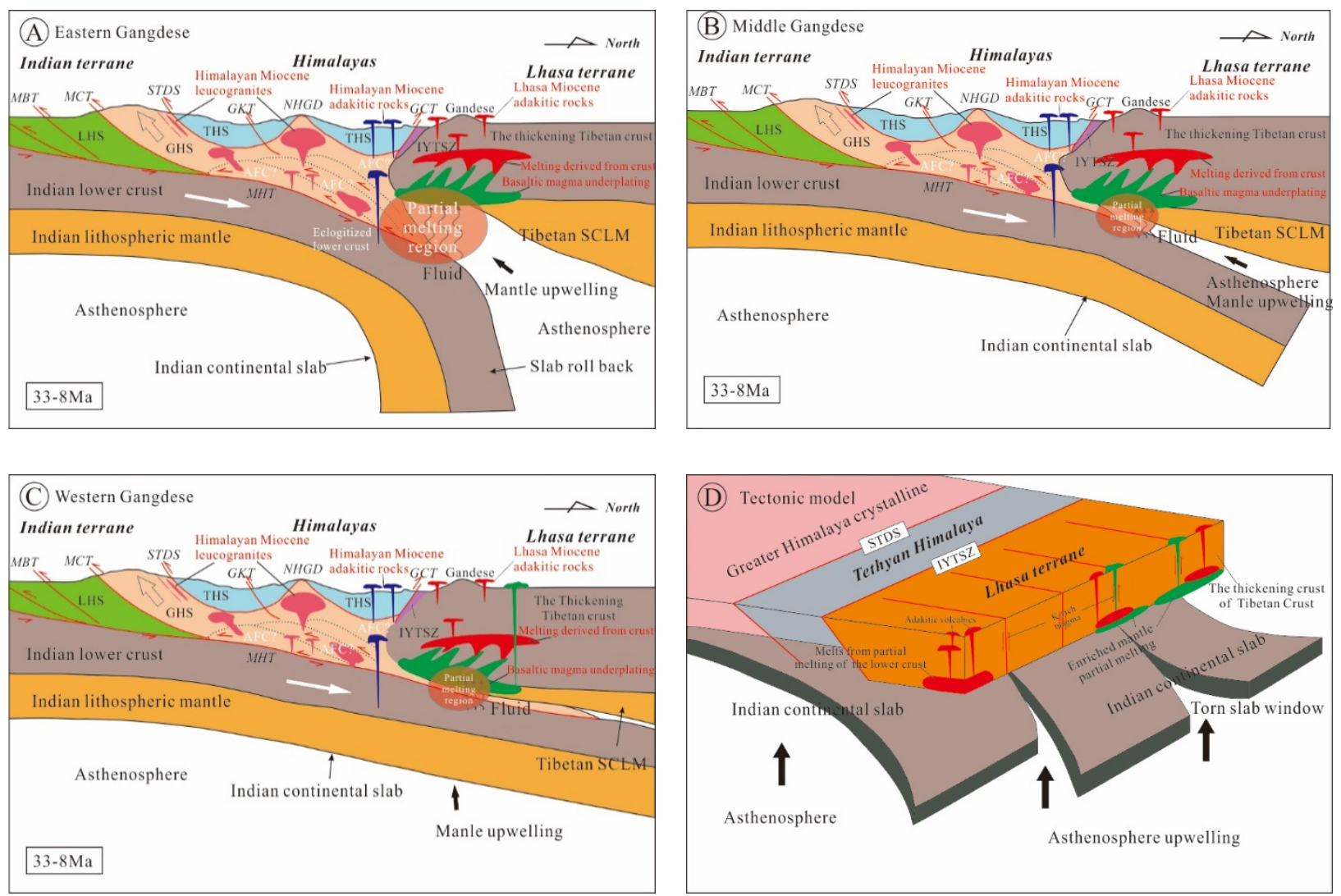

Figure 12. A possible tectonic model for tearing and discordant subduction of the Indian continental slab during the Miocene period. (A) The tectonic model in the eastern Gangdese; (B)The tectonic model in the middle Gangdese; (C)The tectonic model in the western Gangdese; (D) The tectonic model of the southern margin of Gangdese belt. 
After the fragmentation of the Neotethys oceanic crust, the Indian-Eurasian continent continued to subduct northward. As the Indian plate subducted below the Lhasa terrane $[17,77,106,123-126]$, it underwent partial eclogitization and subsequent delamination of the front material of the subducted Indian continental slab [34]. As a result, the front edge of that undergoes tearing, followed by segmented differential subduction. The slab tear zone is similar to a slab window, thereby providing ideal conditions for the upwelling of deep asthenospheric material and mantle-derived magma. With postcollisional stretching and convective thinning of the mantle, asthenosphere materials first undergo thermal upwelling erosion along the subducting slab tear zone and the "slab tear window" caused by the fracture of its boundary. The asthenosphere material rises through the "lamellar tearing window" to provide heat to the upper lithosphere, and the K-rich magma formed by partial melting at the bottom of the lower crust intrudes into the surface along the extensional fissures or remains in the lower crust. These stagnant K-rich mafic magmas locally heated the surrounding lower crust, combined with the release of fluid from the subduction plate and the decompression of extensional structure, which caused the partial melting of the low melting-point components in the lower crust and formed adakitic magmas in post-collisional conditions. In addition, the outcrops of post-collisional potassic-ultrapotassic rocks in the Gangdese belt usually appear in the western part of the structural belt, which reflects the low-angle subduction process of the Indian continental plate in the western part of the structural belt and provides a large amount of material for post-collisional magmatism in the area. However, in the eastern part, due to the high-angle subduction of the Indian plate, the participation of the enriched continental crust material is low, so no large area of contemporaneous potassic-ultrapotassic magma is found.

In conclusion, the Cenozoic Indian Eurasian collision resulted in the shortening of the Gangdese lithosphere and uniform thickening of the crust. With the post-collisional extension and thinning of the lithospheric mantle, the hot asthenosphere beneath the subducted crust was torn along the front edge of the Indian plate and upwelled at the high-angle subduction position of the eastern segment of the plate, resulting in the anatexis of thickened lower crust and forming the main body of Miocene adakitic magmas along the southern margin of the Gangdese belt. The main reason for the difference in material contamination between adakitic magma and the Indian continental plate is that the main body of the magma and the subducted plate are subducted in certain sections.

\section{Conclusions}

This paper selected felsic magmatic rocks in the middle Gangdese as the research object, combining the previous research results data on the adakitic rocks in the southern Gangdese belt of the Lhasa terrane, provide a comprehensive dataset of the geochemistry, zircon $\mathrm{U}-\mathrm{Pb}$ dating, and Sr-Nd (bulk rock) and Hf (zircon) isotopic compositions for the Miocene adakitic rocks formed by post-collision in the south Gangdese belt. These datasets can help us to comprehend the crustal evolution and the dynamic mechanism in the south of the Lhasa terrane. Our key conclusions are summarized as follows:

1. The Tortonian age of the acidic magmatic rocks of the Zongdangcun Formation in the Wuyu Basin in central Gangdese, southern Tibet, is approximately 10.3 $\pm 0.2 \mathrm{Ma}$. These Miocene rocks display the geochemical characteristics of adakites.

2. The analysis of elemental geochemical data and isotope ratios and the estimation of crustal thickness with $\mathrm{Sr} / \mathrm{Y}$ and $(\mathrm{La} / \mathrm{Yb})_{\mathrm{N}}$ values indicate that the post-collisional magmatic rocks in the middle Gangdese belt resulted from the partial melting of the thickened lower crust (essentially formed by the lower crust of the Lhasa block, with a minor contribution from the ancient Indian crust.). The cause of the melting may have been the upwelling of hot asthenospheric material at the bottom of the Indian plate and the lowering of the lower crustal melting curve caused by the infiltration of fluids released from the Indian plate.

3. The bulk-rock Sr-Nd and zircon Hf isotopic compositions of adakitic rocks formed during the post-collisional times reflect the relative depletion of adakitic magma in 
the eastern section and the relative enrichment in the western section of this period. This implies that there may be more lower Indian crust involved in the formation of Miocene adakitic rocks from the east to the west Gangdese belt.

4. The hot asthenosphere beneath the subducted crust tearing along the front edge of the continental Indian plate and upwelling at the high-angle subduction position of the eastern segment of the plate resulted in the thickening of the lower crust and subsequent melting, thereby forming Miocene adakitic magma on the southern margin of the Gangdese belt. The difference in the contribution ratio of the ancient Indian crust in the adakitic magma formed by the segmented subduction plate in the east-west trending segment may be related to the change in the subduction angle of the Indian plate. The tearing and segmented subduction of the front edge of the Indian plate is a possible model for the formation of the post-collisional Gangdese adakitic rocks.

Author Contributions: Conceptualization, H.C. and M.H.; methodology, H.C.; software, F.X.; validation, F.X., G.S. and H.T.; formal analysis, H.C.; investigation, G.S. and H.T.; resources, H.T.; data curation, H.C.; writing—original draft preparation, H.C. and M.H.; writing—review and editing, F.X.; visualization, H.C.; supervision, M.H.; project administration, M.H. All authors have read and agreed to the published version of the manuscript.

Funding: This research received no external funding.

Data Availability Statement: All data used in this work are reported in the main text.

Acknowledgments: We thank James Ogg for his constructive reviews, and helping us with the editing of the English language.

Conflicts of Interest: The authors declare no conflict of interest.

\section{References}

1. Yin, A.; Harrison, T.M. Geologic evolution of the Himalayan-Tibetan orogen. Annu. Rev. Earth Planet. Sci. 2000, 28, 211-280. [CrossRef]

2. Allègre, C.J.; Courtillot, V.; Tapponnier, P.; Hirn, A.; Mattauer, M.; Coulon, C.; Jaeger, J.J.; Achache, J.; Schä, R.U.; Marcoux, J.; et al. Structure and evolution of the Himalaya-Tibet orogenic belt. Nature 1984, 307, 17-22. [CrossRef]

3. Chung, S.L.; Chu, M.F.; Zhang, Y.Q.; Xie, Y.W.; Lo, C.H.; Lee, T.Y.; Lan, C.Y.; Li, X.H.; Zhang, Q.; Wang, Y.Z. Tibetan tectonic evolution inferred from spatial and temporal variations in post-collisional magmatism. Earth Sci. Rev. 2005, 68, 173-196. [CrossRef]

4. Ji, W.Q.; Wu, F.Y.; Chung, S.L.; Li, J.X.; Liu, Z.C. Zircon U-Pb geochronology and Hf isotopic constraints on petrogenesis of the Gangdese batholith, southern Tibet. Chem. Geol. 2009, 262, 229-245. [CrossRef]

5. Zhu, D.C.; Zhao, Z.D.; Niu, Y.L.; Mo, X.X.; Chung, S.L.; Hou, Z.Q.; Wang, L.Q.; Wu, F.Y. The Lhasa terrane: Record of a microcontinent and its histories of drift and growth. Earth Planet. Sci. Lett. 2011, 301, 241-255. [CrossRef]

6. Zhu, D.C.; Zhao, Z.D.; Niu, Y.L.; Dilek, Y.; Hou, Z.Q.; Mo, X.X. The origin and pre-Cenozoic evolution of the Tibetan Plateau. Gondwana Res. 2013, 23, 1429-1454. [CrossRef]

7. Zhu, D.C.; Wang, Q.; Zhao, Z.D.; Chung, S.L.; Cawood, P.A.; Ni, Y.L.; Liu, S.A.; Wu, F.Y.; Mo, X.X. Magmatic record of India-Asia collision. Sci. Rep. 2015, 5, 14289. [CrossRef]

8. Zhu, D.C.; Wang, Q.; Chung, S.L.; Cawood, P.A.; Zhao, Z.D. Gangdese magmatism in southern Tibet and India-Asia convergence since 120 Ma. In Himalayan Tectonics: A Modern Synthesis; Treloar, P.J., Searle, M.P., Eds.; Geological Society; Special Publications: London, UK, 2018; p. 483.

9. Wang, C.; Ding, L.; Zhang, L.Y.; Kapp, P.; Pullen, A.; Yue, Y.H. Petrogenesis of Middle-Late Triassic volcanic rocks from the Gangdese belt, southern Lhasa terrane: Implications for early subduction of Neo-Tethyan oceanic lithosphere. Lithos 2016, 262, 320-333. [CrossRef]

10. Defant, M.J.; Drummond, M.S. Mount St. Helens: Potential example of the partial melting of the subducted lithosphere in a volcanic arc. Geology 1993, 21, 547-550. [CrossRef]

11. Martin, H. Adakitic magmas: Modern analogues of Archaean granitoids. Lithos 1999, 46, 411-429. [CrossRef]

12. Chung, S.L.; Liu, D.Y.; Ji, J.Q.; Chu, M.F.; Lee, H.Y.; Wen, D.J.; Lo, C.H.; Lee, T.Y.; Qian, Q.; Zhang, Q. Adakites from continental collision zones: Melting of thickened lower crust beneath southern Tibet. Geology 2003, 31, 1021-1024. [CrossRef]

13. Hou, Z.Q.; Gao, Y.F.; Qu, X.M.; Rui, Z.Y.; Mo, X.X. Origin ofadakitic intrusives generated during Mid-Miocene east-west extension in southern Tibet. Earth Planet. Sci. Lett. 2004, 220, 139-155. [CrossRef] 
14. Guo, Z.F.; Wilson, M.; Liu, J.Q. Post-collisional adakites in south Tibet: Products of partial melting of subduction-modified lower crust. Lithos 2007, 96, 205-224. [CrossRef]

15. Xu, W.C.; Zhang, H.F.; Guo, L.; Yuan, H.L. Miocene high Sr/Y magmatism, South Tibet: Product of partial melting of subducted Indian continental crust and its tectonic implication. Lithos 2010, 114, 293-306. [CrossRef]

16. Chen, J.L.; Xu, J.F.; Zhao, W.X.; Dong, Y.H.; Wang, B.D.; Kang, Z.Q. Geochemical variations in Miocene adakitic rocks from the western and eastern Lhasa terrane: Implications for lower crustal flow beneath the Southern Tibetan Plateau. Lithos 2011, 125, 928-939. [CrossRef]

17. Zhang, Z.M.; Dong, X.; Xiang, H.X.; Ding, H.X.; He, Z.Y.; Liou, J.G. Reworking of the Gangdese magmatic arc, southeastern Tibet: Postcollisional metamorphism and anatexis. J. Metamorph. Geol. 2015, 33, 1-21. [CrossRef]

18. Yang, Z.M.; Goldfarb, R.; Chang, Z.S. Generation of postcollisional porphyry copper deposits in southern Tibet triggered by subduction of the Indian continental plate. In Tectonics and Metallogeny of the Tethyan Orogenic Belt; Richards, J.P., Ed.; Society of Economic Geologists: Littleton, CO, USA, 2016; Volume 19, pp. 279-300.

19. Zeng, Y.C.; Chen, J.L.; Xu, J.F.; Lei, M.; Xiong, Q.W. Origin of Miocene Cu-bearing porphyries in the Zhunuo region of the southern Lhasa subterrane: Constraints from geochronology and geochemistry. Gondwana Res. 2017, 41, 51-64. [CrossRef]

20. Qu, X.M.; Hou, Z.Q.; Li, Y.G. Melt components derived from a subducted slab in late orogenic ore-bearing porphyries in the Gangdese copper belt, southern Tibetan Plateau. Lithos 2004, 74, 131-148. [CrossRef]

21. Gao, Y.F.; Yang, Z.S.; Santosh, M.; Hou, Z.Q.; Wei, R.H.; Tian, S.H. Adakitic rocks from slabmelt-modified mantle sources in the continental collision zone of southern Tibet. Lithos 2010, 119, 651-663. [CrossRef]

22. Yin, A. Cenozoic tectonic evolution of the Himalayan orogen as constrained by along-strike variation of structural geometry, exhumation history, and foreland sedimentation. Earth Sci. Rev. 2006, 76, 1-131. [CrossRef]

23. Yang, Z.M.; Lu, Y.J.; Hou, Z.Q.; Chang, Z.S. High-Mg diorite from Qulong in southern Tibet: Implications for the genesis of adakite-like intrusions and associated porphyry $\mathrm{Cu}$ deposits in collisional orogens. J. Petrol. 2015, 56, 227-254. [CrossRef]

24. Zhang, Z.M.; Santosh, M. Tectonic evolution of Tibet and surrounding regions. Gondwana Res. 2012, 21, 1-3. [CrossRef]

25. Gehrels, G.; Kapp, P.; De Celles, P.; Pullen, A.; Blakey, R.; Weislogel, A.; Ding, L.; Guynn, J.; Martin, A.; Mc Quarrie, N.; et al. Detrital zircon geochronology of pre-tertiary strata in the Tibetan Himalayan orogen. Tectonics 2011, 30, TC5016. [CrossRef]

26. Zhang, Z.M.; Ding, L.; Zhao, Z.D.; Santosh, M. Tectonic evolution and dynamics of the Tibetan Plateau. Gondwana Res. 2017, 41, 1-8. [CrossRef]

27. Dewey, J.F.; Shackelton, R.M.; Chang, C.F.; Sun, Y.Y. The tectonicevolution of the Tibetan Plateau. Philos. Trans. R. Soc. A Math. Phys. Eng. Sci. 1988, 327, 379-413.

28. Pan, G.T.; Wang, L.Q.; Li, R.S.; Yuan, S.H.; Ji, W.H.; Yin, F.G.; Zhang, W.P.; Wang, B.D. Tectonic evolution of the Qinghai-Tibet Plateau. J. Asian Earth Sci. 2012, 53, 3-14. [CrossRef]

29. Xu, Z.Q.; Wang, Q.; Li, Z.H.; Li, H.Q.; Cai, Z.H.; Liang, F.H.; Dong, H.W.; Cao, H.; Chen, X.J.; Huang, X.M.; et al. Indo-Asian collison: Tectonic transition from compress to strike slip. Acta Geol. Sin. 2016, 90, 1-23, (In Chinese with English abstract).

30. Xu, Z.Q.; Yang, J.S.; Li, H.B.; Ji, S.C.; Zhang, Z.M.; Liu, Y. On the tectonics of the India-Asia collision. Acta Geol. Sin. 2011, 85, 1-33, (In Chinese with English abstract).

31. Hou, Z.Q.; Cook, N.J. Metallogenesis of the Tibetan collisional orogen: A review and introduction to the special issue. Ore Geol. Rev. 2009, 36, 2-24. [CrossRef]

32. Patriat, P.; Achache, J. Indian-Eurasia collision chronology has implications for crustal shortening and driving mechanism of plates. Nature 1984, 311, 615-621. [CrossRef]

33. Klootwijk, C.T.; Gee, J.S.; Peirce, J.W.; Smith, G.M.; McFadden, P.L. An early India-Asia contact: Paleomagnetic constraints from Ninetyeast Ridge, ODP Leg 121. Geology 1992, 20, 395-398. [CrossRef]

34. Leech, M.L.; Singh, S.; Jain, A.K.; Klemperer, S.L.; Manickavasagam, R.M. The onset of India-Asia continental collision: Early, steep subduction required by the timing of UHP metamorphism in the western Himalaya. Earth Planet Sci. Lett. 2005, 234, 83-97. [CrossRef]

35. Zhang, Z.M.; Ding, H.X.; Dong, X.; Tian, Z.L. The Gangdese arc magmatism: From Neo-Tethyan subdction to Indo-Asian collison. Earth Sci. Front. 2018, 25, 078-091, (In Chinese with English abstract).

36. Zhang, Z.M.; Zhao, G.C.; Santosh, M.; Wang, J.L.; Dong, X.; Shen, K. Late Cretaceous charnockite with adakitic afinities from the Gangdese mid-oceanic ridge subduction. Gondwana Res. 2010, 17, 615-631. [CrossRef]

37. Ma, L.; Wang, Q.; Wyman, D.A.; Li, Z.X.; Jiang, Z.Q.; Yang, J.H.; Gou, G.N.; Guo, H.F. Late Cretaceous (100-89 Ma) magnesian charnockites with adakitic affinitiesin the Milin Area, Eastern Gangdese: Partial melting of subducted oceanic crust and implications for crustal growth in Southern Tibet. Lithos 2013, 175/176, 315-332. [CrossRef]

38. Zheng, Y.C.; Hou, Z.Q.; Gong, Y.L.; Liang, W.; Sun, Q.Z.; Zhang, S.; Fu, Q.; Huang, K.X.; Li, Q.Y.; Li, W. Petrogenesis of Cretaceous adakite-like in trusions of the Gangdese Plutonic Belt, southern Tibet: Implications and for mid-ocean ridge subduction and crustal growth. Lithos 2014, 190-191, 240-263. [CrossRef]

39. Weller, O.M.; Stonge, M.R.; Rayner, N.; Searle, M.P.; Waters, D.J. Micoene magmatism in the Western Nyainaentanglha Mountains of southern Tibet: An exhumed bright spot. Lithos 2016, 245, 147-160. [CrossRef]

40. Mo, X.X.; Dong, G.C.; Zhao, Z.D.; Guo, T.Y.; Wang, L.L.; Chen, T. Timing of magma mixing in the Gangdisê magmatic belt during the India-Asian collision: Zircon SHRIMP U-Pb dating. Acta Geol. Sin. 2005, 79, 66-76. 
41. Mo, X.X.; Hou, Z.Q.; Niu, Y.L.; Dong, G.C.; Qu, X.M.; Zhao, Z.D.; Yang, Z.M. Mantle contributions to crustal thickening during continental collision: Evidence from Cenozoic igneous rocks in southern Tibet. Lithos 2007, 96, 225-242. [CrossRef]

42. Chu, M.F.; Chung, S.L.; Song, B.; Liu, D.Y.; O’Reilly, S.Y.; Pearson, N.J.; Ji, J.Q.; Wen, D.J. Zircon U-Pb and Hf isotope constraints on the Mesozoic tectonics and crustal evolution of southern Tibet. Geology 2006, 34, 745-748. [CrossRef]

43. Zhu, D.C.; Zhao, Z.D.; Pan, G.T.; Lee, H.Y.; Kang, Z.Q.; Liao, Z.L.; Wang, L.Q.; Li, G.M.; Dong, G.C.; Liu, B. Early cretaceous subductionrelated adakite-like rocks of the Gangdese Belt, southern Tibet: Products of slab melting and subsequent melt-peridotite interaction? J. Asian Earth Sci. 2009, 34, 298-309. [CrossRef]

44. Turner, S.; Arnaud, N.; Liu, J.; Rogers, N.; Hawkesworth, C.; Harris, N.; Kelley, S.; Van Calsteren, P.; Deng, W. Postcollision, shoshonitic volcanism on the Tibetan Plateau: Implications for convective thinning of the lithosphere and the source of ocean island basalts. J. Petrol. 1996, 37, 45-71. [CrossRef]

45. Miller, C.; Schuster, R.; Klotzli, U.; Frank, W.; Purtscheller, F. Post-collisional potassic and ultrapotassic magmatism in SW Tibet: Geochemical and $\mathrm{Sr}-\mathrm{Nd}-\mathrm{Pb}-\mathrm{O}$ isotopic constraints for mantle source characteristics and petrogenesis. J. Petrol. 1999, 40, 1399-1424. [CrossRef]

46. Williams, H.M.; Turner, S.; Pearce, G.A.; Kelley, S.P.; Harris, N.B.W. Nature of the source regions for post-collisional, potassic magmatism in southern and northern Tibet from geochemical variations and inverse element modeling. J. Petrol. 2004, 45, 555-607. [CrossRef]

47. Zhao, Z.D.; Mo, X.X.; Dilek, Y.; Niu, Y.L.; De Paolo, D.J.; Robinson, P.; Zhu, D.C.; Sun, C.G.; Dong, G.C.; Zhou, S.; et al. Geochemical and $\mathrm{Sr}-\mathrm{Nd}-\mathrm{Pb}-\mathrm{O}$ isotopic compositions of the postcollisional ultrapotassic magmatism in SW Tibet: Petrogenesis and implications for India intra-continental subduction beneath southern Tibet. Lithos 2009, 113, 190-212. [CrossRef]

48. Zheng, Y.C.; Hou, Z.Q.; Li, Q.Y.; Sun, Q.Z.; Liang, W.; Fu, Q.; Li, W.; Huang, K.X. Origin of Late Oligocene adakitic intrusives in the southeastern Lhasa terrane: Evidence from in situ zircon U-Pb dating, $\mathrm{Hf}-\mathrm{O}$ isotopes, and whole-rock geochemistry. Lithos 2012, 148, 296-311. [CrossRef]

49. Sun, C.G.; Zhao, Z.D.; Mo, X.X.; Zhu, D.C.; Dong, G.C.; Zhou, S.; Chen, H.H.; Xie, L.W.; Yang, Y.H.; Sun, J.F.; et al. Enriched mantle source and petrogenesis of Sailipu ultrapotassic rocks in southweatern Tibetan Plateau: Constraints from zircon U-Pb geochronology and Hf isotopic compositions. Acta Petrol. Sin. 2008, 24, 249-264.

50. Gao, Y.F.; Hou, Z.Q.; Kamber, B.S.; Wei, R.H.; Meng, X.J.; Zhao, R.S. Adakite-like porphyries from the southern Tibetan continental collision zones: Evidence for slab melt metasomatism. Contrib. Mineral. Petrol. 2007, 153, 105-120. [CrossRef]

51. Xie, L.W.; Zhan, Y.; Zhnag, H.; Sun, J.; Wu, F. In situ simultaneous determination of trace elements, U-Pb and Lu-Hf isotopes in zircon and baddeleyite. Chin. Sci. Bull. 2008, 53, 1565-1573. [CrossRef]

52. Yuan, H.L.; Gao, S.; Liu, X.M.; Li, H.M.; Gunther, D.; Wu, F.Y. Accurate U-Pb age and trace element determinations of zircon by laser ablation-inductively coupled plasma-mass spectrometry. Geostand. Geoanal. Res. 2004, 28, 353-370. [CrossRef]

53. Liu, Y.; Hu, Z.; Gao, S.; Günther, D.; Xu, J.; Gao, C.; Chen, H. In situ analysis of major and trace elements of anhydrous minerals by LA-ICP-MS without applying an internal standard. Chem. Geol. 2008, 257, 34-43. [CrossRef]

54. Vavra, G. On the kinematics of zircon growth and its petrogenetic significance:a cathodoluminescence study. Contrib. Miner. Pet. 1990, 106, 90-99. [CrossRef]

55. Hanchar, J.M.; Miller, C.F. Zircon zonation patterns as revealed by cathod oluminescence and backscattered electron images: Implications for intepretation of complex crustal histories. Chem. Geol. 1993, 110, 1-13. [CrossRef]

56. Corfu, F.; Hanchar, J.M.; Hoskin, P.W.O.; Kinny, P. Atlas of zircon textures. Rev. Mineral. Geochem. 2003, 53, 469-500. [CrossRef]

57. Wu, Y.B.; Zheng, Y.F. Genesis of zircon and its constraints on interpretation of U-Pb age. Chin. Sci. Bull. 2004, 49, 1554-1569. [CrossRef]

58. Maniar, P.D.; Piccoli, P. Tectonic Discrimination of Granitoids. Geol. Soc. Am. Bull. 1989, 101, 635-643. [CrossRef]

59. Rickwood, P.C. Boundary lines within petrologic diagrams which use oxides of major and minor elements. Lithos 1989, 22, 247-263. [CrossRef]

60. Le Maitre, R.W. Igneous Rocks: A Classification and Glossary of Terms; Cambridge University Press: Cambridge, UK, 2002.

61. Xu, Q.; Zeng, L.S.; Gao, J.H.; Gao, L.; Wang, Y.F.; Hu, Z.P.; Zhao, L.H. Geochemical characteristics and genesis of the Miocene high Sr/Y intermediate-felsic magmatic rocks in eastern Gangdese batholith, southern Tibet. Acta Petrol. Sin. 2019, 35, 1627-1646. [CrossRef]

62. Taylor, S.R.; McLennan, S.M. The Continental Crust: Its Composition and Evolution; Blackwell Scientific Publication: Oxford, UK, $1985 ; 312 \mathrm{p}$.

63. Defant, M.J.; Drummond, M.S. Derivation of some modern arc magmas by melting of young subducted lithosphere. Nature 1990, 347, 662-665. [CrossRef]

64. Kepezhinskas, P.K.; Defant, M.J.; Drummond, M.S. Na metasomatism in the island-arc mantle by slab melt-peridotite interaction: Evidence from mantle xenoliths in the North Kamchatka Arc. J. Petrol. 1995, 36, 1505-1527.

65. Martin, H.; Smithies, R.H.; Rapp, R.; Moyen, J.F.; Champion, D. An Overview of Adakite, Tonalite-Ttrondhjemite-Granodiorite (TTG), and Sanukitoid: Relationships and Some Implications for Crustal Evolution. Lithos 2005, 79, 1-24. [CrossRef]

66. Defant, M.J. Pavel Kepezhinskas. In Evidence Suggests Slab Melting in Arc Magmas; John Wiley \& Sons, Ltd.: Hoboken, NJ, USA, 2001; Volume 82, pp. 65-69.

67. Sun, S.S.; McDonough, W.F. Chemical and isotopic systematics of oceanic basalts: Implications for mantle composition and processes. Geol. Soc. Lond. Spec. Publ. 1989, 42, 313-345. [CrossRef] 
68. Kinny, P.D.; Compston, W.; Williams, I.S. A Reconnaissance Ion-probe Study of Hafnium Isotopes in Zircons. Geochim. Et Cosmochim. Acta 1991, 55, 849-859. [CrossRef]

69. Amelin, Y.; Lee, D.C.; Halliday, A.N.; Pidgeon, R.T. Nature of the Earth's Earliest Crust from Hafnium Isotopes in Single Detrital Zircons. Nature 1999, 399, 252-255. [CrossRef]

70. Vervoort, J.D.; Blichert-Toft, J. Evolution of the depleted mantle: Hf isotope evidence from juvenile rocks through time. Geochim. et Cosmochim. Acta 1999, 63, 533-556. [CrossRef]

71. Leeman, W.P. The influence of crustal structure on compositions of subduction-related magmas. J. Volcanol. Geotherm. Res. 1983, 18, 561-588. [CrossRef]

72. Lee, C.T.A. Copper conundrums. Nat. Geosci. 2014, 7, 10-11. [CrossRef]

73. Farner, M.J.; Lee, C.T.A. Effects of crustal thickness on magmatic differentiation in subduction zone volcanism: A global study. Earth Planet. Sci. Lett. 2017, 470, 96-107. [CrossRef]

74. Chapman, J.B.; Ducea, M.N.; De Celles, P.G.; Profeta, L. Tracking changes in crustal thickness during orogenic evolution with Sr/Y: An example from the North American Cordillera. Geology 2015, 43, 919-922. [CrossRef]

75. Profeta, L.; Ducea, M.N.; Chapman, J.B.; Paterson, S.R.; Gonzales, S.M.H.; Kirsch, M.; Petrescu, L.; De Celles, P.G. Quantifying crustal thickness over time in magmatic arcs. Sci. Rep. 2015, 5, 17786. [CrossRef]

76. Hirn, A.; Lepine, J.C.; Jobert, G.; Sapin, M.; Wittlinger, G.; Xu, Z.X.; Gao, E.Y.; Wang, X.J.; Teng, J.W.; Xiong, S.B.; et al. Crustal structure and variability of the Himalayan border of Tibet. Nature 1984, 307, 23-25. [CrossRef]

77. Nábělek, J.; Hetényi, G.; Vergne, J.; Sapkota, S.; Kafle, B.; Jiang, M.; Su, H.P.; Chen, J.; Huang, B.S.; the Hi-CLIMB Team. Underplating in the Himalaya-Tibet collision zone revealed by the Hi-CLIMB experiment. Science 2009, 325, 1371-1374. [CrossRef]

78. Green, T.H. Experimental studies of trace-element partitioning applicable to igneous petrogenesis-Sedona 16 years later. Chem. Geol. 1994, 117, 1-36. [CrossRef]

79. Foley, S.F.; Jackson, S.E.; Fryer, B.J.; Greenough, J.D.; Jenner, G.A. Trace element partition coefficients for clinopyroxene and phlogopite in an alkaline lamprophyre from Newfoundland by LAM-ICP-MS. Geochim. Cosmochim. Acta 1996, 60, 629-638. [CrossRef]

80. Turner, S.; Hawkesworth, C.; Liu, J.; Rogers, N.; Kelley, S.; Van Calsteren, P. Timing of Tibetan uplift constrained by analysis of volcanic rocks. Nature 1993, 364, 50-54. [CrossRef]

81. Williams, H.M.; Turner, S.; Kelley, S.; Harris, N. Age and composition of dikes in Southern Tibet: New constraints on the timing of east-west extension and its relationship to postcollisional volcanism. Geology 2001, 29, 339-342. [CrossRef]

82. DeCelles, P.G.; Robinson, D.M.; Zandt, G. Implications of shortening in the Himalayan fold-thrust belt for uplift of the Tibetan Plateau. Tectonics 2002, 21, 1062. [CrossRef]

83. Kohn, M.; Parkinson, C.D. Petrologic case for Eocene slab breakoff during the Indo-Asian collision. Geology 2002, 30, 591-594. [CrossRef]

84. Ji, W.Q.; Wu, F.Y.; Chung, S.L.; Wang, X.C.; Liu, C.Z.; Li, Q.L.; Liu, Z.C.; Liu, X.C.; Wang, J.G. Eocene Neo-Tethyan slab breakoff constrained by $45 \mathrm{Ma}$ oceanic island basalt-type magmatism in southern Tibet. Geology 2016, 44, 283-286. [CrossRef]

85. Castillo, P.R.; Janney, P.E.; Solidum, R.U. Petrology and Geochemistry of Camiguin Island, Southern Philippines: Insights to the Source of Adakites and Other Lavas in a Complex Arc Setting. Contrib. Mineral. Petrol. 1999, 134, 33-51. [CrossRef]

86. Macpherson, C.G.; Dreher, S.T.; Thirlwall, M.F. Adakites without slab melting: High pressure differentiation of island arc magma, Mindanao, the Philippines. Earth Planet. Sci. Lett. 2006, 243, 581-593. [CrossRef]

87. Kadioglu, Y.K.; Dilek, Y. Structure and Geochemistry of the Adakitic Horoz Granitoid, Bolkar Mountains, South-central Turkey, and Its Tectonomagmatic Evolution. Int. Geol. Rev. 2010, 52, 505-535. [CrossRef]

88. Stern, R.A.; Hanson, G.N. Archean high-Mg granodiorite: A derivative of light rare earth element-enriched monzodiorite of mantle origin. J. Petrol. 1991, 32, 201-238. [CrossRef]

89. Atherton, M.P.; Petford, N. Generation of sodium-rich magmas from newly underplated basaltic crust. Nature 1993, 362, 144-146. [CrossRef]

90. Xu, J.F.; Shinjo, R.; Defant, M.J.; Wang, Q.; Rapp, R.P. Origin of Mesozoic adakitic intrusive rocks in the Ningzhen area of east China: Partial melting of delaminated lower continental crust? Geology 2002, 30, 1111-1114. [CrossRef]

91. Wang, J.; Hattori, K.H.; Kilian, R.; Stern, C.R. Metasomatism of subarc mantle peridotites below southernmost South America: Reduction of $\mathrm{fO}_{2}$ by slab-melt. Contrib Miner. Pet. 2007, 153, 607-624. [CrossRef]

92. Tatsumi, Y. High-Mg andesites in the Setouchi volcanic belt, Southwestern Japan: Analogy to Archean magmatism and continental crust formation? Annu. Rev. Earth Planet. Sci. 2006, 34, 467-499. [CrossRef]

93. Ding, L.; Kapp, P.; Zhong, D.; Deng, W. Cenozoic volcanism in Tibet: Evidence for a transition from oceanic to continental subduction. J. Petrol. 2003, 44, 1833-1865. [CrossRef]

94. Mo, X.X.; Niu, Y.L.; Dong, G.C.; Zhao, Z.D.; Hou, Z.Q.; Zhou, S.; Ke, S. Contribution of syncollisional felsic magmatism to continental crust growth: A case study of the Paleogene Linzizong volcanic Succession in southern Tibet. Chem. Geol. 2008, 250, 49-67. [CrossRef]

95. Stern, C.R.; Kilian, R. Role of the subducted slab, mantle wedge and continental crust in the generation of adakites from the Andean Austral volcanic zone. Contrib. Mineral. Petrol. 1996, 123, 263-281. [CrossRef]

96. Condie, K.C. TTGs and adakites: Are they both slab melts? Lithos 2005, 80, 33-44. [CrossRef] 
97. Liu, D.; Zhao, Z.; De Paolo, D.J.; Zhu, D.C.; Meng, F.Y.; Shi, Q.S.; Wang, Q. Potassic volcanic rocks and adakitic intrusions in southern Tibet: Insights into mantle-crust interaction and mass transfer from Indian plate. Lithos 2017, 268-271, 48-64. [CrossRef]

98. Gao, Y.F.; Hou, Z.Q.; Wei, R.H.; Meng, X.G.; Yang, Z.S. Eocene high-MgO volcanism in southern Tibet: New constraints for mantle source characteristics and deep processes. Lithos 2008, 105, 63-72. [CrossRef]

99. Sen, C.; Dunn, T. Dehydration melting of a basaltic composition amphibolite at $1.5 \mathrm{GPa}$ and $2.0 \mathrm{GPa}$ Implications for the origin of adakites. Contrib. Mineral. Petrol. 1994, 117, 394-409. [CrossRef]

100. Rapp, R.P.; Watson, E.B. Dehydration melting of metabasalt at 8-32 kbar: Implications for continental growth and crust-mantle recycling. J. Petrol. 1995, 36, 891-931. [CrossRef]

101. Wood, B.J.; Turner, S.P. Origin of primitive high-Mg andesite: Constraints from natural examples and experiments. Earth Planet. Sci. Lett. 2009, 283, 59-66. [CrossRef]

102. Wang, Y.; Deng, T.; Biasatti, D. Ancient diets indicate significant uplift of southern Tibet after ca. 7 Ma. Geology 2006, 34, 309-312. [CrossRef]

103. Jiang, Z.Q.; Wang, Q.; Wyman, D.A.; Tang, G.J.; Jia, X.H.; Yang, Y.H.; Yu, H.X. Origin of 30 Ma Chongmuda adakitic intrusive rocks in the southern Gangdese region, southern Tibet: Partial melting of the northward subducted Indian continent crust? Geochimica 2011, 40, 126-146, (In Chinese with English abstract).

104. Lee, H.Y.; Chung, S.L.; Wang, Y.B.; Zhu, D.C.; Yang, J.H.; Song, B.; Liu, D.Y.; Wu, F.Y. Age, petrogenesis and geological significance of the Linzizong volcanic successions in the Linzhou basin, southern Tibet: Evidence from zircon U-Pb dates and Hf isotopes. Acta Petrol. Sin. 2007, 23, 493-500, (In Chinese with English abstract).

105. Ma, L.; Wang, B.D.; Jiang, Z.Q.; Wang, Q.; Li, Z.X.; Wyman, D.A.; Zhao, S.R.; Yang, J.H.; Gou, G.N.; Guo, H.F. Petrogenesis of the Early Eocene adakitic rocks in the Napuri area, southern Lhasa: Partial melting of thickened lower crust during slab break-off and implications for crustal thickening in southern Tibet. Lithos 2014, 196, 321-338. [CrossRef]

106. Zhang, H.; Harris, N.; Parrish, R.; Kelley, S.; Zhang, L.; Rogers, N.; Argles, T.; King, J. Causes and consequences of protracted melting of the mid-crust exposed in the North Himalayan antiform. Earth Planet. Sci. Lett. 2004, 228, 195-212. [CrossRef]

107. Zeng, L.; Gao, L.; Xie, K.; Hu, G. Concurrence of Mid-Miocene high Sr/Y granite and leucogranite in the Yardoi gneiss dome, Tethyan Himalaya, Southern Tibet. Mineral. Mag. 2011, 75, 2245.

108. Zeng, L.; Gao, L.; Tang, S.; Hou, K.; Guo, C.; Hu, G. Eocene magmatism in the Tethyan Himalaya, southern Tibet. In Tectonics of the Himalaya; Mukherjee, S., Carosi, R., van der Beek, P.A., Mukherjee, B.K., Robinson, D.M., Eds.; Geological Society of London, Special Publications: London, UK, 2015; Volume 412, pp. 287-316. [CrossRef]

109. Owens, T.J.; Zandt, G. Implications of crustal property variations for models of Tibetan Plateau evolution. Nature 1997, $387,37-43$. [CrossRef]

110. Zheng, Y.F.; Zhao, Z.F.; Chen, Y.X. Continental subduction channel processes: Plate interface interaction during continental collision. Chin. Sci. Bull. 2013, 58, 4371-4377. [CrossRef]

111. Chung, S.L.; Chu, M.F.; Ji, J.Q.; O’Reilly, S.Y.; Pearson, N.J.; Liu, D.Y.; Lee, T.Y.; Lo, C.H. The nature and timing of crustal thickening in Southern Tibet. Tectonophysics 2009, 477, 36-48. [CrossRef]

112. Ji, W.Q.; Wu, F.Y.; Liu, C.Z.; Chung, S.L. Early Eocene crustal thickening in southern Tibet: New age and geochemical constraints from the Gangdese batholith. J. Asian Earth Sci. 2012, 53, 82-95. [CrossRef]

113. Chu, M.; Chung, S.; O’Reilly, S.Y.; Pearson, N.J.; Wu, F.; Li, X.; Liu, D.; Ji, J.; Chu, C.; Lee, H. India's hidden inputs to Tibetan orogeny revealed by $\mathrm{Hf}$ isotopes of Transhimalayan zircons and host rocks. Earth Planet. Sci. Lett. 2011, 307, 479-486. [CrossRef]

114. Wang, C.S.; Zhao, X.X.; Liu, Z.F.; Lippert, P.C.; Graham, S.A.; Coe, R.S.; Yi, H.S.; Zhu, L.D.; Liu, S.; Li, Y.L. Constrains on the early uplift history of the Tibetan Plateau. Proc. Natl. Acad. Sci. USA 2008, 105, 4987-4992. [CrossRef]

115. Hou, Z.Q.; Zheng, Y.C.; Yang, Z.M.; Rui, Z.Y.; Zhao, Z.D.; Jiang, S.H.; Qu, X.M.; Sun, Q.Z. Contribution of mantle components within juvenile lower-crust to collisional zone porphyry Cu systems in Tibet. Miner. Depos. 2013, 48, 173-192. [CrossRef]

116. Guo, Z.; Wilson, M.; Zhang, M.; Cheng, Z.; Zhang, L. Post-collisional ultrapotassic mafic magmatism in South Tibet: Products of partial melting of pyroxenite in the mantle wedge induced by roll-back and delamination of the subducted Indian continental lithosphere slab. J. Petrol. 2015, 56, 1365-1406. [CrossRef]

117. Jobert, N.; Journet, B.; Jobert, G.; Hirn, A.; Sun, K.Z. Deep structure of southern Tibet inferred from the dispersion of Rayleigh waves through a long-period seismic network. Nature 1985, 313, 386-388. [CrossRef]

118. Nelson, K.D.; Zhao, W.J.; Brown, L.D.; Kuo, J.; Che, J.K.; Liu, X.W.; Klemperer, S.L.; Makovsky, Y.; Meissner, R.; Mechie, J.; et al. Partially molten middle crust beneath southern Tibet: Synthesis of project INDEPTH results. Science 1996, 274, 1684-1688. [CrossRef] [PubMed]

119. Guan, Q.; Zhu, D.; Zhao, Z.; Dong, G.; Zhang, L.; Li, X.; Liu, M.; Mo, X.; Liu, Y.; Yuan, H. Crustal thickening prior to 38 Ma in southern Tibet: Evidence from lower crust-derived adakitic magmatism in the Gangdese Batholith. Gondwana Res. 2012, 21, 288-299. [CrossRef]

120. Nomade, S.; Renne, P.R.; Mo, X.; Zhao, Z.; Zhou, S. Miocene volcanism in the Lhasa block, Tibet: Spatial trends and geodynamic implications. Earth Planet. Sci. Lett. 2004, 221, 227-243. [CrossRef]

121. Hou, Z.; Zhao, Z.; Gao, Y.; Yang, Z.; Jiang, W. Tearing and dischronal subduction of the Indian continental slab: Evidence from Cenozoic Gangdese volcanomagmatic rocks in south Tibet. Yanshi Xuebao 2006, 22, 761-774.

122. Wen, D.R.; Chung, S.L.; Song, B.; Lizuka, Y.; Yang, H.J.; Ji, J.; Liu, D.; Gallet, S. Late Cretaceous Gangdese intrusions of adakitic geochemical characteristics, SE Tibet: Petrogenesis and tectonic implications. Lithos 2008, 105, 1-11. [CrossRef] 
123. Zhao, W.J.; Nelson, K.D.; Che, J.; Quo, J.; Lu, D.; Wu, C.; Liu, X. Deep seismic reflection evidence for continental underthrusting beneath southern Tibet. Nature 1993, 366, 557-559. [CrossRef]

124. Zhao, W.J.; Kumar, P.; Mechie, J.; Kind, R.; Meissner, R.; Wu, Z.H.; Shi, D.N.; Su, H.P.; Xue, G.Q.; Karplus, M.; et al. Tibetan plate overriding the Asian plate in central and northern Tibet. Nat. Geosci. 2011, 4, 870-873. [CrossRef]

125. Zhao, J.M.; Yuan, X.H.; Liu, H.B.; Kumar, P.; Pei, S.P.; Kind, R.; Zhang, Z.J.; Teng, J.W.; Ding, L.; Gao, X.; et al. The boundary between the Indian and Asian tectonic plates below Tibet. Proc. Natl. Acad. Sci. USA 2010, 107, 11229-11233. [CrossRef] [PubMed]

126. Li, C.; Van der Hilst, R.D.; Meltzer, A.S.; Engdahl, E.R. Subduction of the Indian lithosphere beneath the Tibetan Plateau and Burma. Earth Planet. Sci. Lett. 2008, 274, 157-168. [CrossRef] 